Алгебра и анализ

Tом 22 (2010), № 3
St. Petersburg Math. J.

Vol. 22 (2011), No. 3, Pages 411-435

S 1061-0022(2011)01149-5

Article electronically published on March 17, 2011

\title{
QUANTUM TODA CHAINS INTERTWINED
}

\author{
A. GERASIMOV, D. LEBEDEV, AND S. OBLEZIN \\ To Ludwig Dmitrievich Faddeev on the occasion of his 75th birthday \\ The most convenient model for exploring \\ such relationships is the Toda chain. \\ L. D. Faddeev (Preprint LOMI, P-2-79)
}

\begin{abstract}
An explicit construction of integral operators intertwining various quantum Toda chains is conjectured. Compositions of the intertwining operators provide recursive and $\mathcal{Q}$-operators for quantum Toda chains. In particular the authors' earlier results on Toda chains corresponding to classical Lie algebras are extended to the generic $B C_{n^{-}}$and Inozemtsev-Toda chains. Also, an explicit form of $\mathcal{Q}$-operators is conjectured for the closed Toda chains corresponding to the Lie algebras $B_{\infty}, C_{\infty}$, $D_{\infty}$, the affine Lie algebras $B_{n}^{(1)}, C_{n}^{(1)}, D_{n}^{(1)}, D_{n}^{(2)}, A_{2 n-1}^{(2)}, A_{2 n}^{(2)}$, and the affine analogs of $B C_{n^{-}}$and Inozemtsev-Toda chains.
\end{abstract}

\section{INTRODUCTION}

An interesting integral representation for common eigenfunctions of the quantum $A_{n}$-Toda chain Hamiltonian operators was proposed in Gil. By using the representation theory approach to Toda chain eigenfunctions, this representation was generalized to the other classical series $B_{n}, C_{n}$ and $D_{n}$ GLO2]. In GKLO, GLO2] we stressed an important role of the elementary intertwining operators in the construction of solutions of quantum Toda chains. The common eigenfunctions of the quantum Toda chains corresponding to the same classical series can be obtained by recursive applications of some integral operators. In general, the recursive integral operators can naturally be represented as a product of elementary integral operators intertwining quantum Toda Hamiltonians corresponding to different classical series. The integral kernels of the elementary intertwining operators have a uniform structure of the exponential of a linear combination of exponents in natural coordinates. This decomposition of the recursive operators into elementary intertwiners seems to have a methodological importance for quantization of classical Toda chains. Indeed, for classical Toda chains, the recursive operators are given by complicated canonical transformations. The direct quantization of these canonical transformations is far from obvious. Suppose the classical canonical transformations are split naturally into products of elementary canonical transformations allowing standard quantization. Then the quantization of the canonical transformation

2010 Mathematics Subject Classification. Primary 81Q12.

Key words and phrases. Quantum Toda Hamiltonians, elementary intertwining operator, recursive operator, quantization Pasquier-Gaudin integral $Q$-operator.

Supported by RFBR (grant nos. 08-01-00931-a and 09-01-93108-NCNIL-a). A. Gerasimov was also partly supported by a grant from Science Foundation Ireland. S. Oblezin gratefully acknowledges the support from Deligne's 2004 Balzan prize in mathematics. 
is obtained easily as a product of quantized elementary canonical transformations. As was argued in GLO2 and is demonstrated in this paper, this is precisely the case for Toda chains.

One can expect that the algebra of intertwining operators acting between various Toda chains should have an interesting interpretation in the representation theory of classical series of Lie groups. The representation theory interpretation of a particular instance of the integral intertwining operator commuting with quantum Toda chain Hamiltonians was proposed in GLO3. These operators were identified with the generators of Hecke algebras of the classical Lie groups with respect to maximal compact subgroups. An affine version of these operators was introduced long ago by Pasquier and Gaudin as the $\mathcal{Q}$-operator for the affine Toda chain $[\mathrm{PG}]$.

In this paper we extend the integral intertwiner operator approach to open Toda chains whose representation theory interpretation for generic coupling constants is not known. These include the $B C_{n}$-Toda chains constructed by Sklyanin [S] and the Toda chains proposed by Inozemtsev in the classical setting [I]. In the following we refer to the latter series of Toda chains as the $I_{n}$-Toda chains. We propose elementary intertwining operators for the $B C_{n^{-}}$and $I_{n}$-Toda chains and check the intertwining properties with respect to quadratic quantum Hamiltonians. The elementary intertwiners turn out to relate $B C$ - and $I$-Toda chains, thus stressing the importance of the Inozemtsev-Toda chain series as an honorable member of the Toda chains family. The constructed elementary intertwiners allow us to propose explicit integral expressions for common eigenfunctions of $B C_{n^{-}}$and $I_{n}$-Toda chain Hamiltonians for a generic spectrum.

In the second part of the paper we propose analogs of the Pasquier-Gaudin integral $\mathcal{Q}$-operator $\mathrm{PG}$ for the affine Toda chains based on the classical series of affine Lie algebras $A_{n}^{(1)}, A_{2 n}^{(2)}, A_{2 n-1}^{(2)}, B_{n}^{(1)}, C_{n}^{(1)}, D_{n}^{(1)}, D_{n}^{(2)}$, the infinite Lie algebras $B_{\infty}, C_{\infty}$ and $D_{\infty}$, and the affine and semi-infinite version of $B C_{n^{-}}$and $I_{n}$-Toda chains.

Finally, we emphasize that the explicit integral expressions for common eigenfunctions, constructed by using elementary intertwiners, might provide a general approach to the quantization of classical integrable systems. This program was completely realized for $A_{n}$-Toda chains in GLO3. The first step is a construction of recursive operators as a product of quantized elementary intertwining integral operators and checking commutation relations with quadratic quantum Hamiltonians. The next step is a construction of quadratic Hamiltonian eigenfunctions using the recursive operators. Finally one should check orthogonality and completeness relations for the constructed eigenfunctions. Note that the complete set of eigenfunctions can be regarded (after a standard Fourier transformation) as an integral kernel of a unitary transformation $U$ intertwining quantum Toda chains with the quantum theory of free particles (i.e., having zero potential). The higher quantum Hamiltonians can now be obtained by conjugation by $U$ of the standard set of the higher quantum Hamiltonians for a collection of free particles. The correct classical limit of these Hamiltonians is guaranteed by the correct classical limit of the quantum recursive operators used to construct $U$. It would be interesting to apply this line of reasoning to $B C_{n^{-}}$and $I_{n}$-Toda chains, which do not allow a simple representation theory realization. What is missing at the moment are the orthogonality and completeness relations for these sets of functions. The proof of the orthogonality and completeness relations consists of routine manipulations with iterative integrals and will be published elsewhere. The modern approach to solving quantum integrable systems, based on the quantum inverse scattering method (QISM), achieved a spectacular success for various classes of quantum integrable models (see $[\mathrm{F}]$ for a concise account). One might hope that the approach based on explicit integral operators used in this paper might add some supplementary information about the structure of quantum integrable systems. 
This paper is an updated version of the earlier note GLO1. After [GLO1, GLO2, the paper [KS] with results on classical recursion operators for $B C_{n}$-Toda chains appeared. We expect that the classical limit of our constructions for a $B C_{n}$-Toda chain should be compatible with the results of $\mathrm{KS}$.

The plan of this paper is as follows. In $\S 1$ we summarize the results of [Gi, GKLO, GLO2 for the case of a zero spectrum. In $\S 2$ we propose elementary integral intertwining operators and integral representations for $B C_{n^{-}}$and $I_{n^{-}}$-Toda chain eigenfunctions. In $\S 3$, analogs of the Pasquier-Gaudin $\mathcal{Q}$-operator at zero spectral parameter are proposed for affine versions of the Toda chains corresponding to classical Lie groups and $B C_{n^{-}}$and $I_{n}$-Toda chains. In $\S 4$, similar conjectures are stated for semi-infinite Toda chains.

\section{§1. INTEGRAL REPRESENTATION FOR THE EIGENFUNCTIONS OF QUANTUM TODA CHAINS FOR CLASSICAL GROUPS}

We start with reviewing a construction of the intertwining operators and common eigenfunction of quantum Toda chains corresponding to classical Lie algebras GLO2] Then we propose explicit integral representations of eigenfunctions for generic $B C_{n}$ - and $I_{n}$-Toda chains.

First, we recall the standard facts on Toda chains corresponding to arbitrary root systems (see, e.g., [RSTS]). Let $\mathfrak{g}$ be a simple Lie algebra, $\mathfrak{h}$ a Cartan subalgebra, $n=\operatorname{dim} \mathfrak{h}$ the rank of $\mathfrak{g}, R \subset \mathfrak{h}^{*}$ the root system, and $W$ the Weyl group. We fix a decomposition $R=R_{+} \cup R_{-}$of the roots into positive and negative roots. Let $\alpha_{1}, \ldots, \alpha_{n}$ be the bases of simple roots. Let $($,$) be a W$-invariant bilinear symmetric form on $\mathfrak{h}^{*}$ normalized so that $(\alpha, \alpha)=2$ for a long root. This form provides an identification of $\mathfrak{h}$ with $\mathfrak{h}^{*}$, and thus, can be viewed as a bilinear form on $\mathfrak{h}$. Choose an orthonormal basis $e=\left\{e_{1}, \ldots, e_{n}\right\}$ in $\mathfrak{h}$. Then for any $x \in \mathfrak{h}$ we have a decomposition $x=\sum_{i=1}^{n} x_{i} e_{i}$. With these data, we associate an open Toda chain with a quadratic Hamiltonian

$$
H_{2}^{R}(\underline{x})=-\frac{1}{2} \sum_{i=1}^{n} \frac{\partial^{2}}{\partial x_{i}^{2}}+\sum_{i=1}^{n} g_{i} e^{\alpha_{i}(x)}
$$

where the $g_{i}$ are (in general, complex) coupling constants. This Hamiltonian is one of the generators $\mathcal{H}_{i}^{R}, i=1,2, \ldots, \operatorname{rank}(R)$, of the commutative ring of quantum Hamiltonians of the corresponding Toda chain. The common eigenfunctions of the quantum Hamiltonians satisfy the equations

$$
H_{i}^{R}(\underline{x}) \Psi_{\underline{\lambda}}^{R}(\underline{x})=c_{i}(\underline{\lambda}) \Psi_{\underline{\lambda}}^{R}(\underline{x}),
$$

where the $c_{i}(\underline{\lambda})$ are polynomials in the complex variables $\underline{\lambda}=\left(\lambda_{1}, \ldots, \lambda_{n}\right)$ parametrizing the spectrum. In what follows, we shall consider only the eigenfunctions corresponding to the zero eigenvalues of the Hamiltonians, and omit the index $\underline{\lambda}$. Borrowing physics terminology, we call such eigenfunctions ground state wave functions.

1.1. Integral representation of the eigenfunctions of an $A_{n-1}$-Toda chain. In this subsection we recall a recursive construction [GKLO of the Givental integral representation of the eigenfunctions of a $\mathfrak{g l}_{n}$-Toda chain Gi]. The quantum $\mathfrak{g l}_{n}$-Toda chain is characterized by its linear and quadratic Hamiltonians

$$
\begin{aligned}
& H_{1}^{\mathfrak{g l}}(\underline{x})=\imath \sum_{i=1}^{n} \frac{\partial}{\partial x_{i}}, \\
& H_{2}^{\mathfrak{g l} l_{n}}(\underline{x})=-\frac{1}{2} \sum_{i=1}^{n} \frac{\partial^{2}}{\partial x_{i}^{2}}+\sum_{i=1}^{n-1} g_{i} e^{x_{i+1}-x_{i}},
\end{aligned}
$$


where $\underline{x}=\left(x_{1}, \ldots, x_{n}\right)$ and the $g_{i}$ are coupling constants. These Hamiltonians are the first two among the generators $H_{i}^{\mathfrak{g l}}, i=1,2, \ldots, n$, of the commutative ring of quantum Hamiltonians of the $\mathfrak{g l}_{n}$-Toda chain. The common eigenfunctions of the quantum Hamiltonians satisfy the equations

$$
H_{i}^{\mathfrak{g l} l_{n}}(\underline{x}) \Psi_{\underline{\lambda}}^{\mathfrak{g l} l_{n}}(\underline{x})=c_{i}(\underline{\lambda}) \Psi_{\underline{\lambda}}^{\mathfrak{g l} l_{n}}(\underline{x}),
$$

where the $c_{i}(\underline{\lambda})$ are the symmetric polynomials in the complex variables $\underline{\lambda}=\left(\lambda_{1}, \ldots, \lambda_{n}\right)$ parameterizing the spectrum. For instance, $c_{1}(\underline{\lambda})=\lambda_{1}+\cdots+\lambda_{n}$ and $c_{2}(\underline{\lambda})=\lambda_{1}^{2}+\cdots+\lambda_{n}^{2}$. Common eigenfunctions for the $\mathfrak{s l}_{n}$-Toda chain are obtained by imposing the additional condition $c_{1}(\underline{\lambda})=0$ in (1.3). In the sequel, we shall consider ground state wave functions corresponding to $\underline{\lambda}=0$ and, thus, we shall not distinguish between the $\mathfrak{g l}_{n^{-}}$and $\mathfrak{s l}_{n}$-Toda chains.

1.1.1. The following integral representation for a ground state wave function of the $\mathfrak{g l}_{n}$-Toda chain quantum Hamiltonians was derived in Gi]:

$$
\Psi^{\mathfrak{g l}_{n}}(\underline{x})=\int_{\Gamma} e^{\mathcal{F}_{n}(x)} \prod_{k=1}^{n-1} \prod_{i=1}^{k} d x_{k, i}
$$

where $x_{n, i}:=x_{i}, \underline{x}=\left(x_{1}, \ldots, x_{n}\right)$, the function $\mathcal{F}_{n}(x)$ is given by

$$
\mathcal{F}_{n}(x)=-\sum_{k=1}^{n-1} \sum_{i=1}^{k}\left(e^{x_{k, i}-x_{k+1, i}}+g_{i} e^{x_{k+1, i+1}-x_{k, i}}\right),
$$

and the integration domain $\Gamma$ is a middle-dimensional submanifold in the $n(n-1) / 2$ dimensional complex torus with the coordinates $\left\{\exp x_{k, i}, i \leq 1 \leq k \leq n-1\right\}$. The choice of the integration contour is dictated by the condition on the integrand to decay exponentially at infinities.

The integral representation (1.4) can be derived by using recursion over the rank $n$ of the Lie algebra $\mathfrak{g l}_{n}$ GKLO]. Indeed, (1.4) is naturally represented in the recursive form

$$
\Psi^{\mathfrak{g l}_{n}}(\underline{x})=\int_{\Gamma} \prod_{k=1}^{n-1} Q_{k+1, k}\left(\underline{x}_{k+1} ; \underline{x}_{k}\right) d \underline{x}_{k},
$$

with the integral kernel

$$
Q_{k+1, k}\left(\underline{x}_{k+1} ; \underline{x}_{k}\right)=\exp \left\{-\sum_{i=1}^{k}\left(e^{x_{k, i}-x_{k+1, i}}+g_{i} e^{x_{k+1, i+1}-x_{k, i}}\right)\right\} .
$$

Here we define $\underline{x}_{k}=\left(x_{k, 1}, \ldots, x_{k k}\right)$ and $x_{i}:=x_{n, i}, 1 \leq i \leq n$. The zero eigenvalue property of (1.4) with respect to quantum Hamiltonians is then translated into intertwining relations between the integral operators with the kernels $Q_{k+1, k}$ and the Hamiltonians $H_{i}^{\mathfrak{g l}_{k}}$ and $H_{i}^{\mathfrak{g l}_{k+1}}$. In particular, for the quadratic Hamiltonian (1.2) we have the following easily verified property:

$$
H^{\mathfrak{g l}_{k+1}}\left(\underline{x}_{k+1}\right) Q_{k+1, k}\left(\underline{x}_{k+1}, \underline{x}_{k}\right)=Q_{k+1, k}\left(\underline{x}_{k+1}, \underline{x}_{k}\right) H^{\mathfrak{g l}_{k}}\left(\underline{x}_{k}\right),
$$

where

$$
H^{\mathfrak{g l}_{k}}\left(\underline{x}_{k}\right)=-\frac{1}{2} \sum_{i=1}^{k} \frac{\partial^{2}}{\partial x_{k, i}^{2}}+\sum_{i=1}^{k-1} g_{i} e^{x_{k, i+1}-x_{k, i}} .
$$

Here and in what follows we assume that in the relations similar to (1.8) the Hamiltonian operator on the left-hand side acts on the right and the Hamiltonian on the right-hand 
side acts on the left. From (1.8), we can easily deduce that

$$
\left(-\frac{1}{2} \sum_{i=1}^{n} \frac{\partial^{2}}{\partial x_{i}^{2}}+\sum_{i=1}^{n-1} g_{i} e^{x_{i+1}-x_{i}}\right) \Psi^{\mathfrak{g l}_{n}}(\underline{x})=0 .
$$

The eigenvalue property for higher Hamiltonians is not so easy to verify; it deserves more involved considerations [Gi, GKLO].

1.1.2. The integral operator determined by the kernel (1.7) is closely related to a $\mathcal{Q}$ operator realizing Bäcklund transformations in a Toda chain corresponding to the affine Lie algebra $\hat{\mathfrak{g l}}_{n}$. This is a particular example of the integral intertwining operator introduced by Pasquier and Gaudin [PG]. The integral kernel is given by

$$
Q^{\widehat{\mathfrak{g}}_{n}}(\underline{x}, \underline{y})=\exp \left\{-\sum_{i=1}^{n}\left(e^{x_{i}-y_{i}}+g_{i} e^{y_{i+1}-x_{i}}\right)\right\},
$$

where $x_{i+n}=x_{i}$ and $y_{i+n}=y_{i}$. This operator commutes with the Hamiltonian operators of the closed Toda chain. Thus, for example, for the quadratic Hamiltonian we have

$$
H_{2}^{\hat{\mathfrak{g} l_{n}}}(\underline{x}) Q^{\hat{\mathfrak{g l}}_{n}}(\underline{x}, \underline{y})=Q^{\hat{\mathfrak{g} l_{n}}}(\underline{x}, \underline{y}) H_{2}^{\hat{\mathfrak{g} l_{n}}}(\underline{y}),
$$

where

$$
H_{2}^{\widehat{\mathfrak{g} l_{n}}}(\underline{x})=-\frac{1}{2} \sum_{i=1}^{n} \frac{\partial^{2}}{\partial x_{i}^{2}}+\sum_{i=1}^{n} g_{i} e^{x_{i+1}-x_{i}} .
$$

Here we impose the conditions $x_{i+n}=x_{i}$. The recursive operator (1.7) can be obtained from the operator (1.10) in the limit as $g_{n} \rightarrow 0, x_{n} \rightarrow-\infty$ in such a way that $g_{n} e^{-x_{n}} \rightarrow 0$.

1.2. Toda chains corresponding to classical Lie algebras. In this subsection we recall the explicit form of the quadratic quantum Hamiltonians of Toda chains corresponding to classical Lie algebras.

1.2.1. Let $e=\left\{e_{1}, \ldots, e_{n}\right\}$ be an orthonormal basis in $\mathbb{R}^{n}$. The root system of type $B_{n}$ can be defined as

$$
\alpha_{1}=e_{1}, \quad \alpha_{i+1}=e_{i+1}-e_{i}, \quad 1 \leq i \leq n-1,
$$

and the corresponding Dynkin diagram is

$$
\alpha_{1} \Longleftarrow \alpha_{2}-\cdots-\alpha_{n-1} \longleftarrow \alpha_{n} .
$$

The quadratic Hamiltonian operator of the corresponding Toda chain is given by

$$
H_{2}^{B_{n}}(\underline{x})=-\frac{1}{2} \sum_{i=1}^{n} \frac{\partial^{2}}{\partial x_{i}^{2}}+g_{1} e^{x_{1}}+\sum_{i=1}^{n-1} g_{i+1} e^{x_{i+1}-x_{i}} .
$$

1.2.2. The root system of type $C_{n}$ can be defined as

$$
\alpha_{1}=2 e_{1}, \quad \alpha_{i+1}=e_{i+1}-e_{i}, \quad 1 \leq i \leq n-1,
$$

and the corresponding Dynkin diagram is

$$
\alpha_{1} \Longrightarrow \alpha_{2} \longrightarrow \cdots-\alpha_{n-1} \longrightarrow \alpha_{n} .
$$

The quadratic Hamiltonian operator of the corresponding Toda chain is given by

$$
H^{C_{n}}(\underline{z})=-\frac{1}{2} \sum_{i=1}^{n} \frac{\partial^{2}}{\partial z_{i}^{2}}+2 g_{1} e^{2 z_{1}}+\sum_{i=1}^{n-1} g_{i+1} e^{z_{i+1}-z_{i}},
$$

where $\underline{z}=\left(x_{1}, \ldots, z_{n}\right)$. 
There is a more general Toda chain associated with the root system $C_{n}$ (see, e.g., [RSTS, Remark, p. 61]), with the quadratic Hamiltonian

$$
H_{2}^{B C_{n}^{*}}(\underline{z})=-\frac{1}{2} \sum_{i=1}^{n} \frac{\partial^{2}}{\partial z_{i}^{2}}-\frac{g_{1}}{2} e^{z_{1}}+\frac{g_{1}^{2}}{2} e^{2 z_{1}}+\sum_{i=1}^{n-1} g_{i+1} e^{z_{i+1}-z_{i}} .
$$

The $B C_{n}^{*}$-Toda chain can be obtained as a degeneration of the generic $B C_{n}$-Toda chain discussed in what follows. This explains our notation.

1.2.3. The root system of type $D_{n}$ is

$$
\alpha_{1}=e_{1}+e_{2}, \quad \alpha_{i+1}=e_{i+1}-e_{i}, \quad 1 \leq i \leq n-1,
$$

and the corresponding Dynkin diagram is

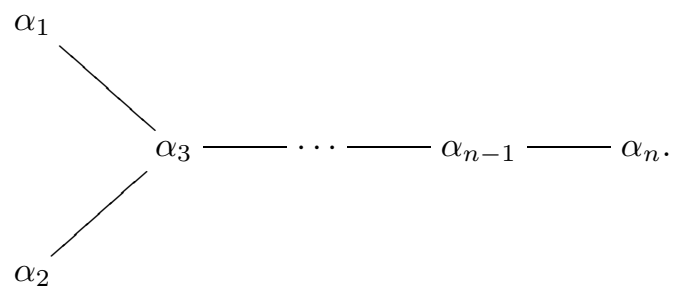

The quadratic Hamiltonian operator of the $D_{n}$-Toda chain is given by

$$
H^{D_{n}}(\underline{x})=-\frac{1}{2} \sum_{i=1}^{n} \frac{\partial^{2}}{\partial x_{i}^{2}}+g_{1} g_{2} e^{x_{1}+x_{2}}+\sum_{i=1}^{n-1} g_{i+1} e^{x_{i+1}-x_{i}}
$$

1.3. Intertwining integral operators. We start with the elementary intertwining operators that relate Toda chains corresponding to different series. These elementary intertwiners have simple integral kernels given by exponentials of linear combinations of exponents. The integration contours are chosen in such a way that the integrals considered below converge.

1.3.1. The integral kernel

$$
Q_{B_{n-1}}^{B C_{n}^{*}}\left(\underline{z}_{n} ; \underline{x}_{n-1}\right)=\exp \left\{-\left(g_{1} e^{z_{n, 1}}+\sum_{i=1}^{n-1}\left(e^{x_{n-1, i}-z_{n, i}}+g_{i+1} e^{z_{n, i+1}-x_{n-1, i}}\right)\right)\right\}
$$

satisfies the relations

$$
H^{B C_{n}^{*}}\left(\underline{z}_{n}\right) \cdot Q_{B_{n-1}}^{B C_{n}^{*}}\left(\underline{z}_{n}, \underline{x}_{n-1}\right)=Q_{B_{n-1}}^{B C_{n}^{*}}\left(\underline{z}_{n}, \underline{x}_{n-1}\right) \cdot H^{B_{n-1}}\left(\underline{x}_{n-1}\right) .
$$

Thus, the corresponding integral operator intertwines the quadratic Hamiltonians of $B C_{n^{-}}^{*}$ and $B_{n-1}$-Toda chains. Similarly, an elementary operator intertwining quadratic $B_{n^{-}}$and $B C_{n}^{*}$-Hamiltonians has an integral kernel

$$
\begin{aligned}
& Q_{B C_{n}^{*}}^{B_{n}}\left(\underline{x}_{n} ; \underline{z}_{n}\right) \\
& \quad=\exp \left\{-\left(g_{1} e^{z_{n, 1}}+\sum_{i=1}^{n-1}\left(e^{x_{n, i}-z_{n, i}}+g_{i+1} e^{z_{n, i+1}-x_{n, i}}\right)+e^{x_{n n}-z_{n n}}\right)\right\} .
\end{aligned}
$$


1.3.2. An integral operator intertwining $C_{n^{-}}$and $D_{n}$-Hamiltonians has the kernel

$$
\begin{aligned}
& Q_{D_{n}}^{C_{n}}\left(\underline{z}_{n} ; \underline{x}_{n}\right) \\
& \quad=\exp \left\{-\left(g_{1} e^{x_{n, 1}+z_{n, 1}}+\sum_{i=1}^{n-1}\left(e^{z_{n, i}-x_{n, i}}+g_{i+1} e^{x_{n, i+1}-z_{n, i}}\right)+e^{z_{n n}-x_{n n}}\right)\right\} .
\end{aligned}
$$

Similarly an integral operator with the kernel

$$
\begin{aligned}
& Q_{C_{n-1}}^{D_{n}}\left(\underline{x}_{n} ; \underline{z}_{n-1}\right) \\
& \quad=\exp \left\{-\left(g_{1} e^{x_{n, 1}+z_{n-1,1}}+\sum_{i=1}^{n-1} e^{z_{n-1, i}-x_{n, i}}+g_{i+1} e^{x_{n, i+1}-z_{n-1, i}}\right)\right\}
\end{aligned}
$$

intertwines the following $D_{n}$ and $C_{n-1}$ quadratic Hamiltonians:

$$
H^{C_{n-1}}\left(\underline{z}_{n-1}\right) \cdot Q_{C_{n-1}}^{D_{n}}\left(\underline{x}_{n} ; \underline{z}_{n-1}\right)=Q_{C_{n-1}}^{D_{n}}\left(\underline{x}_{n} ; \underline{z}_{n-1}\right) \cdot H^{D_{n}}\left(\underline{x}_{n}\right) .
$$

To describe a recursion structure of the ground state eigenfunction of the $C_{n}$-Toda chain, we introduce the integral operators $Q_{C_{k-1}}^{C_{k}}$ with the kernels given for $k=2, \ldots, n$ by convolutions of the intertwining operators $Q_{C_{k}}^{D_{k}}$ and $Q_{D_{k}}^{C_{k-1}}$ :

$$
Q_{C_{k-1}}^{C_{k}}\left(\underline{z}_{k} ; \underline{z}_{k-1}\right)=\int_{\Gamma^{(k)}} Q_{D_{k}}^{C_{k}}\left(\underline{z}_{k} ; \underline{x}_{k}\right) Q_{C_{k-1}}^{D_{k}}\left(\underline{x}_{k} ; \underline{z}_{k-1}\right) d \underline{x}_{k} .
$$

For $k=1$, we set

$$
Q_{C_{0}}^{C_{1}}\left(z_{1,1}\right):=\Psi^{C_{1}}\left(z_{1,1}\right)=\int_{\Gamma^{(1)}} \exp \left\{-g_{1} e^{x_{1,1}+z_{1,1}}-e^{z_{1,1}-x_{1,1}}\right\} d x_{1,1} .
$$

1.3.3. For $k=2, \ldots, n$, we introduce the integral operators $Q_{D_{k-1}}^{D_{k}}$ with the kernels given by convolutions of the kernels of the intertwining operators $Q_{C_{k-1}}^{D_{k}}$ and $Q_{D_{k-1}}^{C_{k-1}}$ :

$$
Q_{D_{k-1}}^{D_{k}}\left(\underline{x}_{k} ; \underline{x}_{k-1}\right)=\int_{\Gamma^{(k)}} Q_{C_{k-1}}^{D_{k}}\left(\underline{x}_{k} ; \underline{z}_{k-1}\right) Q_{D_{k-1}}^{C_{k-1}}\left(\underline{z}_{k-1} ; \underline{x}_{k-1}\right) d \underline{z}_{k-1} .
$$

For $k=1$ we set $Q_{D_{0}}^{D_{1}}\left(x_{1,1}\right)=1$.

1.3.4. Denote $\underline{x}_{k}=\left(x_{k, 1}, \ldots, x_{k, k}\right), \underline{z}_{k}=\left(z_{k, 1}, \ldots, z_{k, k}\right)$. We introduce the integral operators $Q_{B_{k-1}}^{B_{k}}, k=2, \ldots, n$, with the kernels given by the convolutions of the kernels of the elementary intertwiners $Q_{B C_{k}^{*}}^{B_{k}}$ and $Q_{B_{k-1}}^{B C_{k}^{*}}$ :

$$
Q_{B_{k-1}}^{B_{k}}\left(\underline{x}_{k} ; \underline{x}_{k-1}\right)=\int_{\Gamma^{(k)}} Q_{B C_{k}^{*}}^{B_{k}}\left(\underline{x}_{k} ; \underline{z}_{k}\right) Q_{B_{k-1}}^{B C_{k}^{*}}\left(\underline{z}_{k} ; \underline{x}_{k-1}\right) d \underline{z}_{k} .
$$

For $k=1$ we set

$$
Q_{B_{0}}^{B_{1}}\left(x_{1,1}\right):=\Psi^{B_{1}}\left(x_{1,1}\right)=\int_{\Gamma^{(1)}} \exp \left\{-2 g_{1} e^{z_{1,1}}-e^{x_{1,1}-z_{1,1}}\right\} d z_{1,1} .
$$

1.4. Integral representations of Toda chain eigenfunctions. Using the integral intertwining operators discussed above, we can construct recursively ground state eigenfunctions for quantum Toda chains associated with classical Lie algebras. The integral representations obtained in this way are compatible with the representation theory construction of the generic eigenfunctions for classical Lie algebras GLO2. The eigenfunction property with respect to quadratic Hamiltonians follows from the intertwining relations considered in the previous subsection. For higher quantum Hamiltonians, at the moment we should rely on the results obtained in the representation theory approach. 
Proposition 1.1. The ground state eigenfunction for the $B_{n}$-Toda chain has the following integral representation:

$$
\Psi^{B_{n}}\left(x_{1}, \ldots, x_{n}\right)=\int \prod_{\Gamma}{ }_{k=1}^{n} Q_{B_{k-1}}^{B_{k}}\left(\underline{x}_{k} ; \underline{x}_{k-1}\right) \prod_{k=1}^{n-1} d \underline{x}_{k},
$$

where $x_{i}:=x_{n, i}$ and $d \underline{x}_{k}=d x_{k 1} \cdots d x_{k k}$. The eigenfunction is given by the integral over a noncompact domain $\Gamma$ of the real dimension equal to the complex dimension of the generalized flag manifold $X=G / B$, where $G=S O(2 n+1, \mathbb{C}), B$ is a Borel subgroup, and

$$
\sum_{k=1}^{n}(2 k-1)=n^{2}=\left|R_{+}\right|
$$

The domain of integration should be chosen in such a way that the integrand decays exponentially at infinities.

Proposition 1.2. The ground state for the $C_{n}$-Toda chain has the following integral representation:

$$
\Psi^{C_{n}}\left(z_{1}, \ldots, z_{n}\right)=\int \prod_{\Gamma=1}^{n} Q_{C_{k-1}}^{C_{k}}\left(\underline{z}_{k} ; \underline{z}_{k-1}\right) \prod_{k=1}^{n-1} d \underline{z}_{k},
$$

where $z_{i}:=z_{n, i}$ and $d \underline{z}_{k}=d z_{k 1} \cdots d z_{k k}$. The eigenfunction is given by the integral over a noncompact domain $\Gamma$ of real dimension equal to the complex dimension of the generalized flag manifold $X=G / B$, where $G=S O(2 n+1, \mathbb{C}), B$ is a Borel subgroup, and

$$
\sum_{k=1}^{n}(2 k-1)=n^{2}=\left|R_{+}\right|
$$

The domain of integration should be chosen in such a way that the integrand decays exponentially at infinities.

Proposition 1.3. The ground state eigenfunction for the $D_{n}$-Toda chain has the following integral representation:

$$
\Psi^{D_{n}}\left(x_{1}, \ldots, x_{n}\right)=\int \prod_{\Gamma} \prod_{k=1}^{n} Q_{D_{k-1}}^{D_{k}}\left(\underline{x}_{k} ; \underline{x}_{k-1}\right) \prod_{r=1}^{n-1} d \underline{x}_{k},
$$

where $x_{i}:=x_{n, i}$. The eigenfunction is given by the integral over a noncompact domain $\Gamma$ of the real dimension equal to the complex dimension of the generalized flag manifold $X=G / B$, where $G=S O(2 n, \mathbb{C}), B$ is a Borel subgroup, and

$$
\sum_{k=1}^{n-1} 2 k=n(n-1)=\left|R_{+}\right| .
$$

The domain of integration should be chosen in such a way that the integrand decays exponentially at infinities.

1.5. Quadratic Hamiltonians of $B C_{n^{-}}$and $I_{n}$-Toda chains. Here we write out quadratic Hamiltonians of the generic $B C_{n^{-}}$and $I_{n^{-}}$-Toda chains. 
1.5.1. The nonreduced root system of type $B C_{n}$ can be defined as

$$
\alpha_{0}=2 e_{1}, \quad \alpha_{1}=e_{1}, \quad \alpha_{i+1}=e_{i+1}-e_{i}, \quad 1 \leq i \leq n-1,
$$

and the corresponding Dynkin diagram is

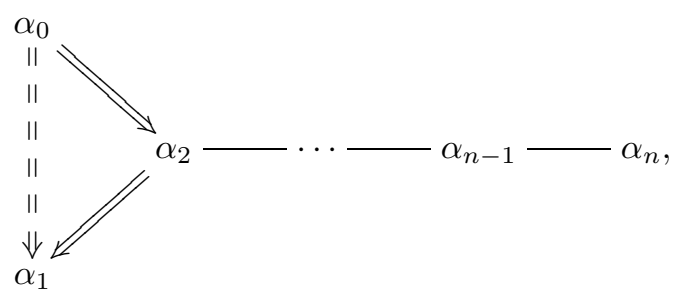

where the two vertices connected by a double dash line correspond to a reduced $\alpha_{1}=e_{1}$ and nonreduced $\alpha_{0}=2 e_{1}=2 \alpha_{1}$ roots.

The corresponding $B C_{n}$-Toda chain has the following quadratic Hamiltonian:

$$
H^{B C_{n}}(\underline{x})=-\frac{1}{2} \sum_{i=1}^{n} \frac{\partial^{2}}{\partial x_{i}^{2}}+g_{1} e^{x_{1}}+g_{2} e^{2 x_{1}}+\sum_{i=1}^{n-1} g_{i+2} e^{x_{i+1}-x_{i}},
$$

where $\underline{x}=\left(x_{1}, \ldots, x_{n}\right)$. Note that the quadratic Hamiltonian (1.12) of the $B C_{n}^{*}$-Toda chain is obtained by the following specialization of the coupling constants: $g_{1}=-g / 2$, $g_{2}=g^{2} / 2$.

1.5.2. The quadratic Hamiltonian of the $I_{n}$-Toda chain [I] is given by

$H^{I_{n}}(\underline{x})=-\frac{1}{2} \sum_{i=1}^{n} \frac{\partial^{2}}{\partial x_{i}^{2}}+\frac{\widetilde{g}_{1}}{\left(e^{x_{1} / 2}-e^{-x_{1} / 2}\right)^{2}}+\frac{\widetilde{g}_{2}}{\left(e^{x_{1}}-e^{-x_{1}}\right)^{2}}+\widetilde{g}_{3} e^{x_{1}+x_{2}}+\sum_{i=1}^{n-1} \widetilde{g}_{i+2} e^{x_{i+1}-x_{i}}$,

where $\underline{x}=\left(x_{1}, \ldots, x_{n}\right)$ and the $g_{i}, i=1, \ldots, n+1$, are generic coupling constants.

1.5.3. The following specialization of the coupling constants $\widetilde{g}_{i}, 1 \leq i \leq n+1$, will be important below:

$$
\widetilde{g}_{1}=-\frac{g_{1}}{\sqrt{2 g_{2}}}, \quad \widetilde{g}_{2}=2 \frac{g_{1}}{\sqrt{2 g_{2}}}\left(\frac{g_{1}}{\sqrt{2 g_{2}}}+1\right), \quad \widetilde{g}_{3}=g_{3} \sqrt{\frac{g_{2}}{2}},
$$

and $\widetilde{g}_{i+2}=g_{i+2}$ for $1<i \leq n-1$. We denote the Hamiltonian with the specialized coupling constants by $H^{I_{n}^{*}}$ :

$$
\begin{aligned}
H^{I_{n}^{*}}\left(\underline{z}_{n}\right)= & -\frac{1}{2} \sum_{i=1}^{n} \frac{\partial^{2}}{\partial z_{n, i}^{2}}-\frac{\frac{g_{1}}{\sqrt{2 g_{2}}}}{\left(e^{-z_{n, 1} / 2}-e^{z_{n, 1} / 2}\right)^{2}}+\frac{2 \frac{g_{1}}{\sqrt{2 g_{2}}}\left(\frac{g_{1}}{\sqrt{2 g_{2}}}+1\right)}{\left(e^{-z_{n, 1}}-e^{z_{n, 1}}\right)^{2}} \\
& +g_{3} \sqrt{\frac{g_{2}}{2}}\left(e^{z_{n, 1}+z_{n, 2}}+e^{z_{n, 2}-z_{n, 1}}\right)+\sum_{i=2}^{n-1} g_{i+2} e^{z_{n, i+1}-z_{n, i}}
\end{aligned}
$$

1.6. Integral intertwining operators. Now we propose the $B C_{n^{-}}$and $I_{n^{-}}$analogs of the intertwining integral operators. The intertwining relations with quadratic quantum Hamiltonians are checked straightforwardly. Hopefully, similar intertwining relations hold true for higher quantum Hamiltonians. 
Lemma 1.1. The integral operator determined by the kernel

$$
\begin{aligned}
& Q_{I_{n}^{*}}^{B C_{n}}\left(\underline{x}_{n}, \underline{z}_{n}\right)=\left(\frac{1-e^{z_{n, 1}}}{1+e^{z_{n, 1}}}\right)^{\frac{g_{1}}{\sqrt{2 g_{2}}}} \\
& \quad \times \exp \left\{-\left(\sqrt{\frac{g_{2}}{2}}\left(e^{x_{n, 1}+z_{n, 1}}+e^{x_{n, 1}-z_{n, 1}}\right)+\sum_{i=2}^{n} g_{i+1} e^{z_{n, i}-x_{n, i-1}}+e^{x_{n, i}-z_{n, i}}\right)\right\}
\end{aligned}
$$

intertwines the quadratic $B C_{n}$ - and $I_{n}^{*}$-Hamiltonians (1.26) and (1.27),

$$
H^{B C_{n}}\left(\underline{x}_{n}\right) \cdot Q_{I_{n}^{*}}^{B C_{n}}\left(\underline{x}_{n}, \underline{z}_{n}\right)=Q_{I_{n}^{*}}^{B C_{n}}\left(\underline{x}_{n}, \underline{z}_{n}\right) \cdot H^{I_{n}^{*}}\left(\underline{z}_{n}\right) .
$$

Lemma 1.2. Let $Q_{I_{n+1}^{*}}^{B C_{n}}$ be the integral operator with the kernel

$$
\begin{aligned}
Q_{I_{n+1}^{*}}^{B C_{n}}\left(\underline{x}_{n}, \underline{z}_{n+1}\right)= & \left(\frac{1-e^{z_{n+1,1}}}{1+e^{z_{n+1,1}}}\right)^{\frac{g_{1}}{\sqrt{2 g_{2}}}} \exp \left\{-\left(\sqrt{\frac{g_{2}}{2}}\left(e^{x_{n, 1}+z_{n+1,1}}+e^{x_{n, 1}-z_{n+1,1}}\right)\right.\right. \\
& \left.\left.+\sum_{i=2}^{n}\left(g_{i+1} e^{z_{n+1, i}-x_{n, i-1}}+e^{x_{n, i}-z_{n+1, i}}\right)+g_{n+2} e^{z_{n+1, n+1}-x_{n n}}\right)\right\} .
\end{aligned}
$$

The following intertwining relation between the quadratic quantum $B C_{n}$ - and $I_{n+1}^{*}$ Hamiltonians is valid:

$$
H^{B C_{n}}\left(\underline{x}_{n}\right) \cdot Q_{I_{n+1}^{*}}^{B C_{n}}\left(\underline{x}_{n}, \underline{z}_{n+1}\right)=Q_{I_{n+1}^{*}}^{B C_{n}}\left(\underline{x}_{n}, \underline{z}_{n+1}\right) \cdot H^{I_{n+1}^{*}}\left(\underline{z}_{n+1}\right) .
$$

We denote the kernel of the inverse integral transformation by

$$
Q_{B C_{n}}^{I_{n+1}^{*}}\left(\underline{z}_{n+1}, \underline{x}_{n}\right):=Q_{I_{n+1}^{*}}^{B C_{n}}\left(\underline{x}_{n}, \underline{z}_{n+1}\right) .
$$

For any $k=2, \ldots, n$, we define integral operators $Q_{B C_{k}}^{B C_{k-1}}$ as convolutions of the kernels of the elementary intertwiners $Q_{I_{k}^{*}}^{B C_{k}}$ and $Q_{B C_{k-1}}^{I_{k}^{*}}$ :

$$
Q_{B C_{k-1}}^{B C_{k}}\left(\underline{x}_{k} ; \underline{x}_{k-1}\right)=\int_{\Gamma^{(k)}} Q_{I_{k}^{*}}^{B C_{k}}\left(\underline{x}_{k} ; \underline{z}_{k}\right) Q_{B C_{k-1}}^{I_{k}^{*}}\left(\underline{z}_{k} ; \underline{x}_{k-1}\right) d \underline{z}_{k} .
$$

For $k=1$ we set

$$
Q_{B C_{0}}^{B C_{1}}\left(x_{1,1}\right)=\int_{\Gamma^{(1)}}\left(\frac{1-e^{z_{11}}}{1+e^{z_{11}}}\right)^{\frac{2 g_{1}}{\sqrt{2 g_{2}}}} \exp \left\{-\sqrt{g_{2} / 2}\left(e^{x_{11}+z_{11}}+e^{x_{11}-z_{11}}\right)\right\} d z_{11} .
$$

Also, we introduce the following quadratic $B C$ - and $I$-Hamiltonians:

$$
\begin{aligned}
H^{B C_{n}}(\underline{x})= & -\frac{1}{2} \sum_{i=1}^{n} \frac{\partial^{2}}{\partial x_{i}^{2}}+g_{1} e^{x_{1}}+g_{2} e^{2 x_{1}}+\sum_{k=1}^{n-1} g_{k+2} e^{x_{k+1}-x_{k}}, \\
H^{I_{n}}(\underline{z})= & -\frac{1}{2} \sum_{i=1}^{n} \frac{\partial^{2}}{\partial z_{i}^{2}}+\frac{\widetilde{g}_{1}}{\left(e^{-z_{1} / 2}-e^{z_{1} / 2}\right)^{2}}+\frac{\widetilde{g}_{2}}{\left(e^{-z_{1}}-e^{z_{1}}\right)^{2}} \\
& +g_{3} \sqrt{\frac{g_{2}}{2}}\left(e^{z_{1}+z_{2}}+e^{z_{2}-z_{1}}\right)+\sum_{k=2}^{n-1} g_{k+2} e^{z_{k+1}-z_{k}},
\end{aligned}
$$

where

$$
\widetilde{g}_{1}=-\frac{(2 \imath a+1) g_{1}}{\sqrt{2 g_{2}}}, \quad \tilde{g}_{2}=2\left(\imath a+\frac{g_{1}}{\sqrt{2 g_{2}}}\right)+2\left(\imath a+\frac{g_{1}}{\sqrt{2 g_{2}}}\right)^{2} .
$$

Note that the coupling constants $\widetilde{g}_{1}$ and $\widetilde{g}_{2}$ are generic, provided the parameter $a$ is generic. 
Proposition 1.4. The integral operator with the kernel

$$
Q_{B C_{n}}^{I_{n}}\left(\underline{z}_{n} ; \underline{x}_{n} \mid a\right)=\left(1-e^{2 z_{n, 1}}\right)^{-2 \imath a} \cdot \exp \left\{\imath a \sum_{i=1}^{n}\left(z_{n, i}-x_{n, i}\right)\right\} Q_{B C_{n}}^{I_{n}^{*}}\left(\underline{z}_{n} ; \underline{x}_{n}\right)
$$

intertwines the quadratic Hamiltonians of $I_{n^{-}}$and $B C_{n}$-type Toda chains,

$$
H^{I_{n}}\left(\underline{z}_{n}\right) \cdot Q_{B C_{n}}^{I_{n}}\left(\underline{z}_{n} ; \underline{x}_{n} \mid a\right)=Q_{B C_{n}}^{I_{n}}\left(\underline{z}_{n} ; \underline{x}_{n} \mid a\right) \cdot H^{B C_{n}}\left(\underline{x}_{n}\right) .
$$

Proposition 1.5. The integral operator with the kernel

$$
Q_{B C_{n}}^{I_{n+1}}\left(\underline{z}_{n+1} ; \underline{x}_{n}\right)=\left(1-e^{2 z_{n+1,1}}\right)^{-2 \imath a} \cdot \exp \left\{\imath a\left(\sum_{i=1}^{n+1} z_{n+1, i}-\sum_{i=1}^{n} x_{n, i}\right)\right\} \cdot Q_{B C_{n}}^{I_{n+1}^{*}}\left(\underline{z}_{n} ; \underline{x}_{n}\right)
$$

intertwines the Hamiltonians $H^{I_{n+1}}\left(\underline{z}_{n+1}\right)-\frac{a^{2}}{2}$ and $H^{B C_{n}}\left(\underline{x}_{n}\right)$, i.e.,

$$
\left(H^{I_{n+1}}\left(\underline{z}_{n+1}\right)-\frac{a^{2}}{2}\right) \cdot Q_{B C_{n}}^{I_{n+1}}\left(\underline{z}_{n+1} ; \underline{x}_{n} \mid a\right)=Q_{B C_{n}}^{I_{n+1}}\left(\underline{z}_{n+1} ; \underline{x}_{n} \mid a\right) \cdot H^{B C_{n}}\left(\underline{x}_{n}\right) .
$$

The kernel of the inverse integral transformation is denoted by

$$
Q_{I_{k}}^{B C_{k}}\left(\underline{x}_{k}, \underline{z}_{k} \mid a\right):=Q_{B C_{k}}^{I_{k}}\left(\underline{z}_{k}, \underline{x}_{k} \mid a\right) .
$$

In what follows, we introduce three types of iterative kernels. For any $k=2, \ldots, n$, we define integral operators $Q_{B C_{k}}^{B C_{k-1}}$ depending on an auxiliary parameter as convolutions of the kernels of the elementary intertwiners $Q_{I_{k}}^{B C_{k}}$ and $Q_{B C_{k-1}}^{I_{k}}$ :

$$
Q_{B C_{k-1}}^{B C_{k}}\left(\underline{x}_{k} ; \underline{x}_{k-1} \mid \lambda_{k}\right)=\int_{\Gamma^{(k)}} Q_{I_{k}}^{B C_{k}}\left(\underline{x}_{k} ; \underline{z}_{k} \mid \lambda_{k}\right) Q_{B C_{k-1}}^{I_{k}}\left(\underline{z}_{k} ; \underline{x}_{k-1} \mid \lambda_{k}\right) d \underline{z}_{k} .
$$

For $k=1$ we set

$$
\begin{aligned}
Q_{B C_{0}}^{B C_{1}}\left(x_{1,1} \mid \lambda_{1}\right)= & \int_{\Gamma^{(1)}}\left(1-e^{2 z_{11}}\right)^{-2 \imath \lambda_{1}} e^{\imath \lambda_{1} z_{11}}\left(\frac{1-e^{z_{11}}}{1+e^{z_{11}}}\right)^{\frac{2 g_{1}}{\sqrt{2 g_{2}}}} \\
& \times \exp \left\{-\sqrt{g_{2} / 2}\left(e^{x_{11}+z_{11}}+e^{x_{11}-z_{11}}\right)\right\} d z_{11} .
\end{aligned}
$$

For $k=2, \ldots, n$, we introduce the integral operators $Q_{I_{k}}^{I_{k+1}}$,

$$
Q_{I_{k-1}}^{I_{k}}\left(\underline{z}_{k} ; \underline{z}_{k-1}\right)=\int_{\Gamma^{(k)}} Q_{B C_{k-1}}^{I_{k}}\left(\underline{z}_{k} ; \underline{x}_{k-1} \mid a\right) Q_{I_{k-1}}^{B C_{k-1}}\left(\underline{x}_{k-1} ; \underline{z}_{k-1} \mid a\right) d \underline{x}_{k-1} .
$$

For $k=1$ we take

$$
Q_{I_{0}}^{I_{1}}\left(z_{11}\right)=\left(1+e^{z_{11}}\right)^{-\frac{g_{1}}{\sqrt{2 g_{2}}}-\imath a}\left(1-e^{z_{11}}\right)^{\frac{g_{1}}{\sqrt{2 g_{2}}}-\imath a} e^{\imath a z_{11}} .
$$

Now, for $k=2, \ldots, n$, we introduce the integral operators $\Lambda_{I_{k}}^{I_{k+1}}$ :

$$
\begin{aligned}
\Lambda_{I_{k-1}}^{I_{k}} & \left(\underline{z}_{k}, \underline{z}_{k-1} \mid \lambda_{k}\right)=\int_{\Gamma^{(1)}}\left(Q_{B C_{k}}^{I_{k}}\left(\underline{z}_{k} ; \underline{x}_{k} \mid a\right) Q_{I_{k}}^{B C_{k}}\left(\underline{x}_{k} ; \underline{u}_{k} \mid \lambda_{k}\right)\right) \\
& \times\left(Q_{B C_{k-1}}^{I_{k}}\left(\underline{u}_{k} ; \underline{x}_{k-1} \mid \lambda_{k}\right) Q_{I_{k-1}}^{B C_{k-1}}\left(\underline{x}_{k-1} ; \underline{u}_{k-1} \mid \lambda_{k}\right)\right) \\
& \times\left(Q_{B C_{k-1}}^{I_{k-1}}\left(\underline{u}_{k-1} ; \underline{x}_{k-1} \mid \lambda_{k}\right) Q_{I_{k-1}}^{B C_{k-1}}\left(\underline{x}_{k-1} ; \underline{z}_{k-1} \mid a\right)\right) d \underline{x}_{k} d \underline{x}_{k-1} d \underline{u}_{k} d \underline{u}_{k-1} .
\end{aligned}
$$

For $k=1$,

$$
\Lambda_{I_{0}}^{I_{1}}\left(z_{11} \mid \lambda_{1}\right)=\int d x_{11} d u_{11} Q_{B C_{1}}^{I_{1}}\left(z_{11}, x_{11} \mid a\right) Q_{I_{1}}^{B C_{1}}\left(x_{11}, u_{11} \mid \lambda_{1}\right) Q_{B C_{0}}^{I_{1}}\left(u_{11} \mid \lambda_{1}\right),
$$

where

$$
Q_{B C_{0}}^{I_{1}}\left(u_{11} \mid \lambda_{1}\right)=\left(1+e^{u_{11}}\right)^{-\frac{g_{1}}{\sqrt{2 g_{2}}}-\imath \lambda_{1}}\left(1-e^{u_{11}}\right)^{\frac{g_{1}}{\sqrt{2 g_{2}}}-\imath \lambda_{1}} e^{\imath \lambda_{1} u_{11}} .
$$


1.7. Givental representations for the eigenfunctions of $B C_{n^{-}}$and $I_{n}$-Toda chains. In this section we conjecture integral representations for elementary operators that intertwine the $I_{n}$-Toda chain Hamiltonians with generic coupling constants and the $B C_{n}$-Toda chain Hamiltonians with generic coupling constants. By using intertwining relations we introduce the conjectural integral formula for the eigenfunction of the $I_{n}$-Toda chain with generic coupling constants corresponding to zero eigenvalues.

Conjecture 1.1. The ground state eigenfunction for the $B C_{n}$-Toda chain has the following integral representation:

$$
\Psi^{B C_{n}}\left(x_{1}, \ldots, x_{n}\right)=\int \prod_{\Gamma=1}^{n} Q_{B C_{k-1}}^{B C_{k}}\left(\underline{x}_{k} ; \underline{x}_{k-1}\right) \prod_{k=1}^{n-1} d \underline{x}_{k},
$$

where $x_{i}:=x_{n, i}$. The domain of integration $\Gamma$ should be chosen in such a way that the integrand decays exponentially at infinities.

This conjecture is supported by various degenerations to be considered in the next section. Also, it is easily verified for quadratic Hamiltonians by using the intertwining relations (1.28), (1.29).

Conjecture 1.2. The eigenfunction for the $B C_{n}$-Toda chain corresponding to the generic eigenvalues parametrized by $\underline{\lambda}=\left(\lambda_{1}, \ldots, \lambda_{n}\right)$ is given by

$$
\Psi_{\underline{\lambda}}^{B C_{n}}\left(x_{1}, \ldots, x_{n}\right)=\int \prod_{\Gamma=1}^{n} Q_{B C_{k-1}}^{B C_{k}}\left(\underline{x}_{k} ; \underline{x}_{k-1} \mid \lambda_{k}\right) \prod_{k=1}^{n-1} d \underline{x}_{k},
$$

where $x_{i}:=x_{n, i}$. The domain of integration $\Gamma$ should be chosen in such a way that the integrand decays exponentially at infinities.

It is easy to check this conjecture for the quadratic Hamiltonians with the help of the following iterative procedure:

$$
H^{B C_{k}}\left(\underline{x}_{k}\right) \cdot Q_{B C_{k-1}}^{B C_{k}}\left(\underline{x}_{k} ; \underline{x}_{k-1} \mid \lambda_{k}\right)=Q_{B C_{k-1}}^{B C_{k}}\left(\underline{x}_{k} ; \underline{x}_{k-1} \mid \lambda_{k}\right) \cdot\left(H^{B C_{k-1}}\left(\underline{x}_{k-1}\right)+\frac{\lambda^{2}}{2}\right) .
$$

At the last step of the iteration we should check the relation

$$
H^{B C_{1}}\left(x_{11}\right) \Psi_{\lambda_{1}}^{B C_{1}}\left(x_{11}\right)=\frac{\lambda_{1}^{2}}{2} \Psi_{\lambda_{1}}^{B C_{1}}\left(x_{11}\right)
$$

where

$$
\begin{aligned}
& \Psi_{\lambda_{1}}^{B C_{1}}\left(x_{11}\right)=Q_{B C_{0}}^{B C_{1}}\left(x_{11}\right) \\
& \quad=\int_{\Gamma^{(1)}}\left(1-e^{2 z_{11}}\right)^{-2 \imath \lambda_{1}}\left(\frac{1-e^{z_{11}}}{1+e^{z_{11}}}\right)^{\frac{2 g_{1}}{\sqrt{2 g_{2}}}} \exp \left\{-\sqrt{g_{2} / 2}\left(e^{x_{11}+z_{11}}+e^{x_{11}-z_{11}}\right)\right\} d z_{11} .
\end{aligned}
$$

The last statement can be verified directly. Notice that the particular cases $g_{1}=0$ and $g_{2}=0$ corresponding to $B_{n^{-}}$and $C_{n}$-Toda chains, respectively, can be transformed (up to a certain multiplier depending on the spectrum) to the corresponding eigenfuctions with nonzero spectrum given in GLO2.

Conjecture 1.3. The eigenfunction for the $I_{n}$-Toda chain with generic coupling constants given by (1.30)-(1.31), corresponding to the eigenvalues parametrized by $\underline{\lambda}=$ $\left(\lambda_{1}, \ldots, \lambda_{n}\right)$, has the integral representation

$$
\Psi_{\lambda_{1}, \ldots, \lambda_{n}}^{I_{n}}\left(z_{1}, \ldots, z_{n}\right)=\int \prod_{\Gamma=1}^{n} \Lambda_{I_{k-1}}^{I_{k}}\left(\underline{z}_{k} ; \underline{z}_{k-1} \mid \lambda_{k}\right) \prod_{k=1}^{n-1} d \underline{z}_{k},
$$

where $\Lambda_{I_{k-1}}^{I_{k}}\left(\underline{z}_{k} ; \underline{z}_{k-1} \mid \lambda_{k}\right)$ is defined by (1.34) and $z_{i}:=z_{n, i}$. The domain of integration $\Gamma$ should be chosen in such a way that the integrand decays exponentially at infinities. 
This conjecture is easily verified for the quadratic Hamiltonian by using iteratively the relations

$$
H^{I_{k}}\left(\underline{z}_{k}\right) \cdot \Lambda_{I_{k-1}}^{I_{k}}\left(\underline{z}_{k} ; \underline{z}_{k-1}\right)=\Lambda_{I_{k-1}}^{I_{k}}\left(\underline{z}_{k} ; \underline{z}_{k-1}\right) \cdot\left(H^{I_{k-1}}\left(\underline{z}_{k-1}\right)+\frac{\lambda_{k}^{2}}{2}\right)
$$

and the identity

$$
\left(H^{I_{1}}\left(u_{11}\right)-\frac{\lambda^{2}}{2}\right) Q_{B C_{0}}^{I_{1}}\left(u_{11} \mid \lambda_{1}\right)=0
$$

1.7.1. Various limiting cases of the conjectures above are compatible with the results presented in GLO2 and recalled in the preceding section. Consider the specialization at $a=0$ of the integral kernel $Q_{B C_{n}}^{I_{n}}$ :

$$
\begin{aligned}
& Q_{B C_{n}}^{I_{n}^{*}}\left(\underline{z}_{n} ; \underline{x}_{n}\right):=\left[Q_{B C_{n}}^{I_{n}}\left(\underline{z}_{n} ; \underline{x}_{n}\right)\right]_{a=0}=\left(\frac{1-e^{z_{n, 1}}}{1+e^{z_{n, 1}}}\right)^{\frac{g_{1}}{\sqrt{2 g_{2}}}} \\
& \quad \times \exp \left\{-\sqrt{\frac{g_{2}}{2}}\left(e^{x_{n, 1}+z_{n, 1}}+e^{x_{n, 1}-z_{n, 1}}\right)-\sum_{k=1}^{n-1} g_{k+2} e^{z_{n, k+1}-x_{n, k}}+e^{x_{n, k+1}-z_{n, k+1}}\right\},
\end{aligned}
$$

which intertwines the $B C_{n}$-Toda chain quadratic Hamiltonian

$$
H^{B C_{n}}\left(\underline{x}_{n}\right)=-\frac{1}{2} \sum_{i=1}^{n} \frac{\partial^{2}}{\partial x_{n, i}^{2}}+g_{1} e^{x_{n, 1}}+g_{2} e^{2 x_{n, 1}}+\sum_{k=1}^{n-1} g_{k+2} e^{x_{n, k+1}-x_{n, k}}
$$

with the specialized $I_{n}$-Toda chain quadratic Hamiltonian

$$
\begin{aligned}
H^{I_{n}^{*}}\left(\underline{z}_{n}\right)= & -\frac{1}{2} \sum_{i=1}^{n} \frac{\partial^{2}}{\partial z_{n, i}^{2}}+\frac{\tilde{g}_{1}}{\left(e^{-z_{n, 1} / 2}-e^{z_{n, 1} / 2}\right)^{2}}+\frac{\widetilde{g}_{2}}{\left(e^{-z_{n, 1}}-e^{z_{n, 1}}\right)^{2}} \\
& +g_{3} \sqrt{\frac{g_{2}}{2}}\left(e^{z_{n, 1}+z_{n, 2}}+e^{z_{n, 2}-z_{n, 1}}\right)+\sum_{k=2}^{n-1} g_{k+2} e^{z_{n, k+1}-z_{n, k}}
\end{aligned}
$$

where

$$
\widetilde{g}_{1}=-\frac{g_{1}}{\sqrt{2 g_{2}}}, \quad \widetilde{g}_{2}=2\left(\frac{g_{1}}{\sqrt{2 g_{2}}}\right)\left(\frac{g_{1}}{\sqrt{2 g_{2}}}+1\right) .
$$

The Hamiltonian $H^{I_{n}^{*}}(\underline{z})$ is not generic, because its coupling constants $\widetilde{g}_{1}$ and $\widetilde{g}_{2}$ only depend on the combination $\frac{g_{1}}{\sqrt{2 g_{2}}}$. Shifting the variable $z_{1} \rightarrow z_{1}+\frac{1}{2} \ln \frac{g_{2}}{2}$, we obtain the following expressions for the specialized $H^{I_{n}^{*}}\left(\underline{z}_{n}\right)$ and $Q_{B C_{n}}^{I_{n}^{*}}\left(\underline{z}_{n} ; \underline{x}_{n}\right)$ when $a=0$ (below, the tildes over $H^{I_{n}^{*}}$ and $Q_{I_{n}^{*}}^{B C_{n}}$ denote an application of the shift of the variable $\left.z_{1}\right)$ :

$$
\begin{aligned}
\tilde{H}^{I_{n}^{*}}\left(\underline{z}_{n}\right)= & -\frac{1}{2} \sum_{i=1}^{n} \frac{\partial^{2}}{\partial z_{n, i}^{2}}-\frac{g_{1}}{2} \frac{e^{-z_{n, 1}}+\frac{g_{2}}{2} e^{z_{n, 1}}-g_{1}}{\left(e^{-z_{n, 1}}-\frac{g_{2}}{2} e^{z_{n, 1}}\right)^{2}} \\
& +\frac{g_{2} g_{3}}{2} e^{z_{n, 1}+z_{n, 2}}+\sum_{i=1}^{n-1} g_{i+2} e^{z_{n, i+1}-z_{n, i}}, \\
\widetilde{Q}_{B C_{n}}^{I_{n}^{*}}\left(\underline{z}_{n}, \underline{x}_{n}\right)= & \left(\frac{1-\sqrt{\frac{g_{2}}{2}} e^{z_{n, 1}}}{1+\sqrt{\frac{g_{2}}{2}} e^{z_{n, 1}}}\right)^{\frac{g_{1}}{\sqrt{2 g_{2}}}} \exp \left\{-\left(\frac{g_{2}}{2} e^{x_{n, 1}+z_{n, 1}}+e^{x_{n, 1}-z_{n, 1}}\right.\right. \\
& \left.\left.+\sum_{k=1}^{n-1} g_{k+2} e^{z_{n, k+1}-x_{n, k}}+e^{x_{n, k+1}-z_{n, k+1}}\right)\right\} .
\end{aligned}
$$


The following limiting relations among various Toda chains can be verified directly.

(1) As $g_{1} \rightarrow 0$, we have

$$
\begin{aligned}
& \lim _{g_{1} \rightarrow 0} H^{B C_{n}}\left(\underline{x}_{n}\right)=H^{C_{n}}\left(\underline{x}_{n}\right), \quad \lim _{g_{1} \rightarrow 0} \widetilde{H}^{I_{n}^{*}}\left(\underline{z}_{n}\right)=H^{D_{n}}\left(\underline{z}_{n}\right), \\
& \lim _{g_{1} \rightarrow 0} \widetilde{Q}_{B C_{n}}^{I_{n}^{*}}\left(\underline{z}_{n}, \underline{x}_{n}\right)=Q_{C_{n}}^{D_{n}}\left(\underline{z}_{n}, \underline{x}_{n}\right) .
\end{aligned}
$$

(2) As $g_{2} \rightarrow 0$, we obtain

$$
\begin{aligned}
& \lim _{g_{2} \rightarrow 0} H^{B C_{n}}\left(\underline{x}_{n}\right)=H^{B_{n}}\left(\underline{x}_{n}\right), \quad \lim _{g_{2} \rightarrow 0} \widetilde{H}^{I_{n}^{*}}\left(\underline{z}_{n}\right)=H^{B C_{n}^{*}}\left(\underline{z}_{n}\right), \\
& \lim _{g_{2} \rightarrow 0} \widetilde{Q}_{B C_{n}}^{I_{n}^{*}}\left(\underline{z}_{n}, \underline{x}_{n}\right)=Q_{B_{n}}^{B C_{n}^{*}}\left(\underline{z}_{n}, \underline{x}_{n}\right) .
\end{aligned}
$$

The last formula stems readily from the following simple observation:

$$
\lim _{\varepsilon \rightarrow 0} \frac{g}{\sqrt{2 \varepsilon}} \ln \frac{1-\sqrt{\frac{\varepsilon}{2}} e^{z}}{1+\sqrt{\frac{\varepsilon}{2}} e^{z}}=\lim _{\varepsilon \rightarrow 0} \frac{g}{\sqrt{2 \varepsilon}}\left(-2 \sqrt{\frac{\varepsilon}{2}} e^{z}+o(\varepsilon)\right)=-g e^{z} .
$$

\section{§2. INTERTWINERS FOR AFFInE TODA CHAins}

In this section we generalize the construction of elementary intertwiners to the classical series of affine Lie algebras. For the necessary facts in the theory of affine Lie algebras, we refer the reader to $[\mathrm{K}]$. First, we recall the construction of the $Q$-operator for the $A_{n}^{(1)}$ Toda chain [PG]. Using the elementary intertwiners, we propose integral operators that intertwine affine Toda chains corresponding to affine Lie algebras of the same rank.

2.1. Quadratic Hamiltonians of affine Toda chains. We start with the list of quadratic Hamiltonians of affine Toda chains considered below.

2.1.1. The simple roots for the system of type $A_{n}^{(1)}$ in the standard basis $\left\{e_{1}, \ldots, e_{n}\right\}$ are as follows:

$$
\alpha_{0}=e_{1}-e_{n+1}, \quad \alpha_{i}=e_{i+1}-e_{i}, \quad 1 \leq i \leq n .
$$

The corresponding Dynkin diagram is

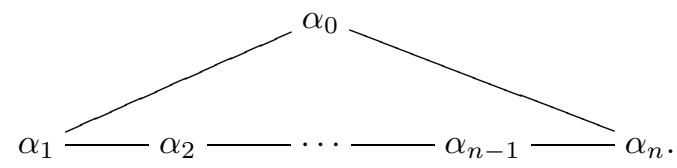

The quadratic Hamiltonian operator for the $A_{n}^{(1)}$ closed Toda chain looks like this:

$$
\mathcal{H}^{A_{n}^{(1)}}\left(\underline{x}_{n+1}\right)=-\frac{1}{2} \sum_{i=1}^{n+1} \frac{\partial^{2}}{\partial x_{n+1, i}^{2}}+g_{1} e^{x_{n+1,1}-x_{n+1, n+1}}+\sum_{i=1}^{n} g_{i+1} e^{x_{n+1, i+1}-x_{n+1, i}} .
$$

2.1.2. The simple roots of the twisted affine root system $A_{2 n}^{(2)}$ can be expressed in terms of the standard basis $\left\{e_{i}\right\}$ as follows:

$$
\begin{aligned}
\alpha_{1}=e_{1}, \quad \alpha_{i+1} & =e_{i+1}-e_{i}, \quad 1 \leq i \leq n-1, \\
\alpha_{n+1} & =-2 e_{n} .
\end{aligned}
$$

The corresponding Dynkin diagram is given by

$$
\alpha_{1} \Longleftarrow \alpha_{2}-\cdots-\alpha_{n-1} \Longleftarrow \alpha_{n} .
$$


We introduce the affine nonreduced root system $B C_{n}^{\prime}$ with simple roots given by

$$
\begin{aligned}
\alpha_{0}=2 e_{1}, \quad \alpha_{1}=e_{1}, \quad \alpha_{i+1} & =e_{i+1}-e_{i}, \quad 1 \leq i \leq n-1, \\
\alpha_{n+1} & =-e_{n}-e_{n-1}
\end{aligned}
$$

the corresponding Dynkin diagram is

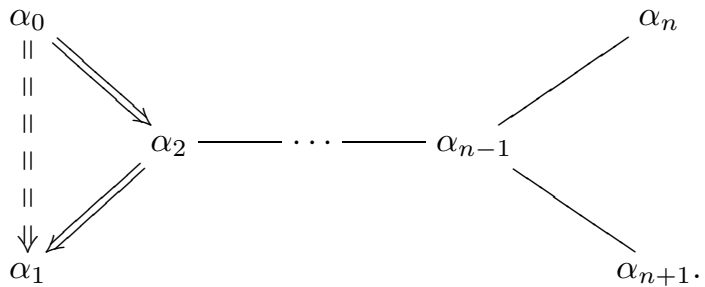

2.1.3. The simple roots of the twisted affine root system $A_{2 n-1}^{(2)}$ are given by

$$
\begin{aligned}
\alpha_{1}=2 e_{1}, \quad \alpha_{i+1} & =e_{i+1}-e_{i}, \quad 1 \leq i \leq n-1, \\
\alpha_{n+1} & =-e_{n}-e_{n-1},
\end{aligned}
$$

and the corresponding Dynkin diagram is

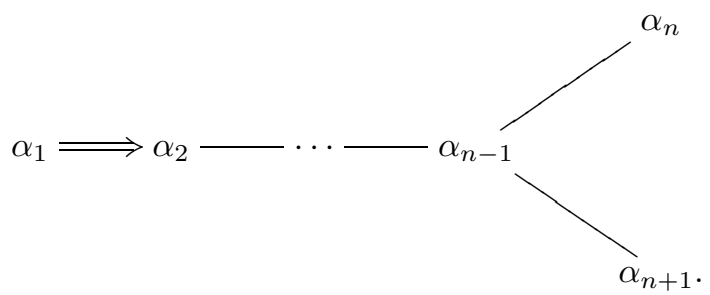

2.1.4. The simple roots of the affine root system $B_{n}^{(1)}$ are given by

$$
\begin{aligned}
\alpha_{1}=e_{1}, \quad \alpha_{i+1} & =e_{i+1}-e_{i}, \quad 1 \leq i \leq n-1, \\
\alpha_{n+1} & =-e_{n}-e_{n-1},
\end{aligned}
$$

and the corresponding Dynkin diagram is

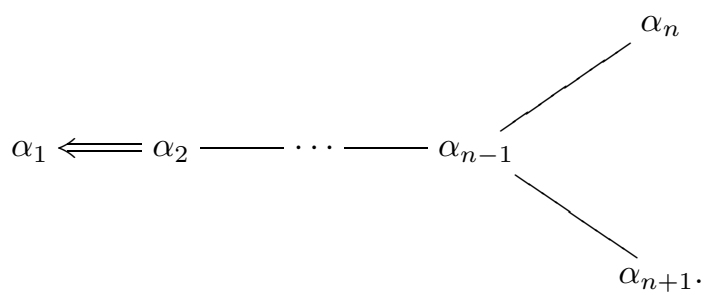

2.1.5. We introduce the affine nonreduced root system $B C_{n}^{\prime \prime}$ with simple roots given by

$$
\begin{aligned}
\alpha_{0}=2 e_{1}, \quad \alpha_{1}=e_{1}, \quad \alpha_{i+1} & =e_{i+1}-e_{i}, \quad 1 \leq i \leq n-1, \\
\alpha_{n+1} & =-2 e_{n} ;
\end{aligned}
$$


then the corresponding Dynkin diagram is given by

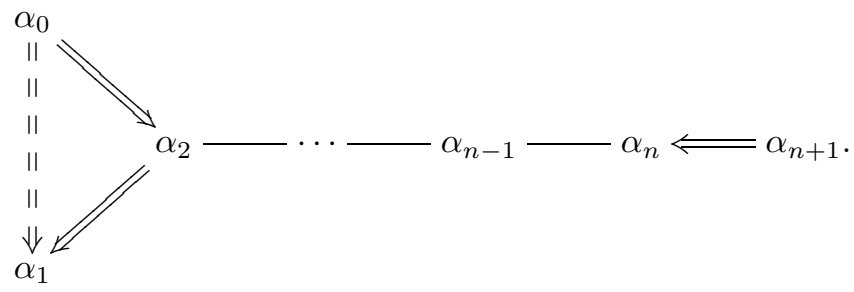

2.1.6. The simple roots of the affine root system $C_{n}^{(1)}$ are

$$
\begin{aligned}
\alpha_{1}=2 e_{1}, \quad \alpha_{i+1} & =e_{i+1}-e_{i}, \quad 1 \leq i \leq n-1, \\
\alpha_{n+1} & =-2 e_{n},
\end{aligned}
$$

and the corresponding Dynkin diagram is

$$
\alpha_{1} \Longrightarrow \alpha_{2} \longrightarrow \cdots-\alpha_{n-1} \longrightarrow \alpha_{n} \Longleftarrow \alpha_{n+1} .
$$

2.1.7. The simple roots of the affine root system $D_{n}^{(1)}$ are

$$
\begin{aligned}
\alpha_{1}=e_{1}+e_{2}, \quad \alpha_{i+1} & =e_{i+1}-e_{i}, \quad 1 \leq i \leq n-1, \\
\alpha_{n+1} & =-e_{n}-e_{n-1},
\end{aligned}
$$

and the corresponding Dynkin diagram is

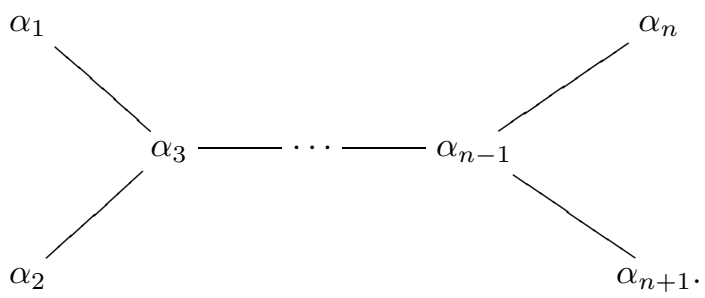

2.1.8. The simple roots of the affine root system $D_{n}^{(2)}$ are

$$
\begin{aligned}
\alpha_{1}=e_{1}, \quad \alpha_{i} & =e_{i+1}-e_{i}, \quad 1 \leq i \leq n-1, \\
\alpha_{n+1} & =-e_{n},
\end{aligned}
$$

and the corresponding Dynkin diagram is given by

$$
\alpha_{1} \Longleftarrow \alpha_{1} \longrightarrow \cdots-\alpha_{n} \Longrightarrow \alpha_{n+1} \text {. }
$$

2.1.9. The simple roots of the (nonreduced) affine root system $\widehat{B C}_{n+1}$ are as follows:

$$
\begin{aligned}
\alpha_{0} & =2 e_{1}, \quad \alpha_{1}=e_{1}, \\
\alpha_{i+1} & =e_{i+1}-e_{i}, \quad 1 \leq i \leq n, \\
\alpha_{n+2} & =-e_{n+1}, \quad \alpha_{n+3}=-2 e_{n+1},
\end{aligned}
$$

and the corresponding Dynkin diagram is given by

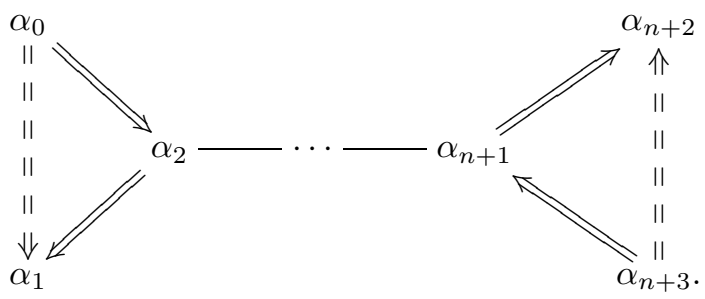


The generic quadratic Toda Hamiltonian associated with the root system $\widehat{B C}_{n}$, see (2.1), looks like this:

$$
\begin{aligned}
\mathcal{H}^{\widehat{B C}}\left(\underline{x}_{n}\right)= & -\frac{1}{2} \sum_{i=1}^{n} \frac{\partial^{2}}{\partial x_{n, i}^{2}}+g_{1} e^{x_{n, 1}}+g_{2} e^{2 x_{n, 1}} \\
& +\sum_{i=1}^{n-1} g_{i+2} e^{x_{n, i+1}-x_{n, i}}+g_{n+2} e^{-2 x_{n n}}+g_{n+3} e^{-x_{n n}} .
\end{aligned}
$$

2.2. Elementary intertwiners. In $[\mathrm{PG}]$, Pasquier and Gaudin introduced the integral $\mathcal{Q}$-operator determined by the integral kernel

$$
Q^{A_{n}^{(1)}}\left(x_{1}, \ldots, x_{n+1} ; y_{1}, \ldots, y_{n+1}\right)=\exp \left\{-\sum_{i=1}^{n+1}\left(e^{x_{i}-y_{i}}+g_{i+1} e^{y_{i+1}-x_{i}}\right)\right\},
$$

where $y_{n+2}=y_{1}, g_{n+2}=g_{1}$. In accordance with [PG], this integral kernel satisfies the intertwining relations

$$
\mathcal{H}^{A_{n}^{(1)}}\left(\underline{x}_{n+1}\right) \cdot Q^{A_{n}^{(1)}}\left(\underline{x}_{n+1} ; \underline{y}_{n+1}\right)=Q^{A_{n}^{(1)}}\left(\underline{x}_{n+1} ; \underline{y}_{n+1}\right) \cdot \mathcal{H}^{A_{n}^{(1)}}\left(\underline{y}_{n+1}\right) .
$$

Below we list kernels of the integral operators that conjecturally intertwine the Hamiltonians of the affine Toda chains corresponding to other classical series of (twisted) affine Lie algebras. We checked these intertwining relations against the quadratic Hamiltonians.

2.2.1. The integral operator with the kernel

$$
\begin{array}{r}
Q_{B C_{n+1}^{\prime}}^{A_{2 n}^{(2)}}\left(\underline{x}_{n}, \underline{z}_{n+1}\right)=\exp \left\{-g_{1} e^{z_{n+1,1}}-\sum_{i=1}^{n}\left(e^{x_{n, i}-z_{n+1, i}}+g_{i+1} e^{z_{n+1, i+1}-x_{n, i}}\right)\right. \\
\left.-g_{n+2} e^{-z_{n+1, n+1}-x_{n n}}\right\}
\end{array}
$$

intertwines the Hamiltonian operators for the $A_{2 n}^{(2)}$ - and $B C_{n+1}^{(2)}$-Toda chains:

$$
\begin{aligned}
\mathcal{H}^{A_{2 n}^{(2)}}\left(\underline{x}_{n}\right)= & -\frac{1}{2} \sum_{i=1}^{n} \frac{\partial^{2}}{\partial x_{n, i}^{2}}+g_{1} e^{x_{n, 1}}+\sum_{i=1}^{n-1} g_{i+1} e^{x_{n, i+1}-x_{n, i}}+2 g_{n+1} g_{n+2} e^{-2 x_{n n}}, \\
\widetilde{\mathcal{H}}^{B C_{n+1}^{\prime}}\left(\underline{z}_{n+1}\right)= & -\frac{1}{2} \sum_{i=1}^{n+1} \frac{\partial^{2}}{\partial z_{n+1, i}^{2}}-\frac{g_{1}}{2} e^{z_{n+1,1}}+\frac{g_{1}^{2}}{2} e^{2 z_{n+1,1}} \\
& +\sum_{i=1}^{n} g_{i+1} e^{z_{n+1, i+1}-z_{n+1, i}}+g_{n+2} e^{-z_{n+1, n+1}-z_{n+1, n}} .
\end{aligned}
$$

The elementary intertwiner (2.2) is involutive; we use the following notation for the integral kernel of the inverse transformation:

$$
Q_{A_{2 n}^{(2)}}^{B C_{n+1}^{\prime}}\left(\underline{z}_{n+1}, \underline{x}_{n}\right):=Q_{B C_{n+1}^{\prime}}^{A_{2 n}^{(2)}}\left(\underline{x}_{n}, \underline{z}_{n+1}\right) .
$$

2.2.2. The integral operator represented by the kernel

$$
\begin{aligned}
& Q_{A_{2 n-1}^{(2)}}^{A_{(2)}^{(2)}}\left(\underline{x}_{n}, \underline{z}_{n}\right)=\exp \left\{-g_{1} e^{x_{n, 1}+z_{n, 1}}-\sum_{i=1}^{n-1}\left(e^{x_{n, i}-z_{n, i}}+g_{i+1} e^{z_{n, i+1}-x_{n, i}}\right)\right. \\
& \left.-e^{x_{n n}-z_{n n}}-g_{n+1} e^{-x_{n n}-z_{n n}}\right\}
\end{aligned}
$$


intertwines the Hamiltonian operators for the $A_{2 n-1}^{(2)}$-Toda chains with generic coupling constants:

$$
\begin{aligned}
& \mathcal{H}^{A_{2 n-1}^{(2)}}\left(\underline{x}_{n}\right)=-\frac{1}{2} \sum_{i=1}^{n} \frac{\partial^{2}}{\partial x_{n, i}^{2}}+2 g_{1} e^{2 x_{n, 1}}+\sum_{i=1}^{n-1} g_{i+1} e^{x_{n, i+1}-x_{n, i}}+g_{n} g_{n+1} e^{-x_{n n}-x_{n, n-1}}, \\
& \widetilde{\mathcal{H}}^{A_{2 n-1}^{(2)}}\left(\underline{z}_{n}\right)=-\frac{1}{2} \sum_{i=1}^{n} \frac{\partial^{2}}{\partial z_{n, i}^{2}}+g_{1} g_{2} e^{z_{n, 1}+z_{n, 2}}+\sum_{i=1}^{n-1} g_{i+1} e^{z_{n, i+1}-z_{n, i}}+2 g_{n+1} e^{-2 z_{n n}} .
\end{aligned}
$$

In what follows we use a separate notation for the integral kernel of the inverse transformation:

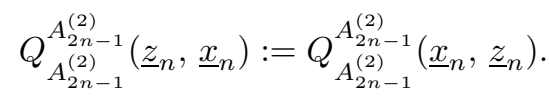

2.2.3. The integral operator represented by the kernel

$$
\left.\begin{array}{r}
Q_{B C_{n}^{\prime \prime}}^{B_{n}^{(1)}}\left(\underline{x}_{n}, \underline{z}_{n}\right)=\exp \left\{-g_{1} e^{z_{n, 1}}-\sum_{i=1}^{n-1}\left(e^{x_{n, i}-z_{n, i}}+g_{i+1} e^{z_{n, i+1}-x_{n, i}}\right)\right. \\
-e^{x_{n n}-z_{n n}}-g_{n+1} e^{-x_{n n}-z_{n n}}
\end{array}\right\}
$$

intertwines the Hamiltonians of the $B_{n}^{(1)}$ - and $B C_{n}^{\prime \prime}$-Toda chains:

$$
\begin{aligned}
& \mathcal{H}^{B_{n}^{(1)}}\left(\underline{x}_{n}\right)=-\frac{1}{2} \sum_{i=1}^{n} \frac{\partial^{2}}{\partial x_{n, i}^{2}}+g_{1} e^{x_{n, 1}}+\sum_{i=1}^{n-1} g_{i+1} e^{x_{n, i+1}-x_{n, i}}+g_{n} g_{n+1} e^{-x_{n n}-x_{n, n-1}}, \\
& \tilde{\mathcal{H}}^{B C_{n}^{\prime \prime}}\left(\underline{z}_{n}\right)=-\frac{1}{2} \sum_{i=1}^{n} \frac{\partial^{2}}{\partial z_{n, i}^{2}}-\frac{g_{1}}{2} e^{z_{n, 1}}+\frac{g_{1}^{2}}{2} e^{2 z_{n, 1}}+\sum_{i=1}^{n-1} g_{i+1} e^{z_{n, i+1}-z_{n, i}}+2 g_{n+1} e^{-2 z_{n n}} .
\end{aligned}
$$

We use the following notation for the integral kernel of the inverse transformation:

$$
Q_{B_{n}^{(1)}}^{B C_{n}^{\prime \prime}}\left(\underline{z}_{n}, \underline{x}_{n}\right):=Q_{B C_{n}^{\prime \prime}}^{B_{n}^{(1)}}\left(\underline{x}_{n}, \underline{z}_{n}\right)
$$

2.2.4. The integral operator with the kernel

$$
\begin{aligned}
Q_{D_{n+1}^{(1)}}^{C_{n}^{(1)}}\left(\underline{x}_{n}, \underline{z}_{n+1}\right)=\exp \left\{-g_{1} e^{x_{n, 1}+z_{n+1,1}}\right. \\
\left.\quad-\sum_{i=1}^{n}\left(e^{x_{n, i}-z_{n+1, i}}+g_{i+1} e^{z_{n+1, i+1}-x_{n, i}}\right)-g_{n+2} e^{-z_{n+1, n+1}-x_{n n}}\right\}
\end{aligned}
$$

intertwines the Hamiltonian operators for the $C_{n}^{(1)}$ - and $D_{n+1}^{(1)}$-Toda chains:

$$
\begin{gathered}
\mathcal{H}^{C_{n}^{(1)}}\left(\underline{x}_{n}\right)=-\frac{1}{2} \sum_{i=1}^{n} \frac{\partial^{2}}{\partial x_{n, i}^{2}}+2 g_{1} e^{2 x_{n, 1}}+\sum_{i=1}^{n-1} g_{i+1} e^{x_{n, i+1}-x_{n, i}}+2 g_{n+1} g_{n+2} e^{-2 x_{n n}}, \\
\mathcal{H}^{D_{n+1}^{(1)}\left(\underline{z}_{n+1}\right)=-} \frac{1}{2} \sum_{i=1}^{n+1} \frac{\partial^{2}}{\partial z_{n+1, i}^{2}}+g_{1} g_{2} e^{z_{n+1,1}+z_{n+1,2}} \\
+\sum_{i=1}^{n} g_{i+1} e^{z_{n+1, i+1}-z_{n+1, i}}+g_{n+2} e^{-z_{n+1, n+1}-z_{n+1, n}} .
\end{gathered}
$$


2.2.5. The integral operator with the kernel

$$
\begin{aligned}
& Q_{C_{n-1}^{(1)}}^{D_{(1)}^{(1)}}\left(\underline{x}_{n}, \underline{z}_{n-1}\right)=\exp \left\{-g_{1} e^{x_{n, 1}+z_{n-1,1}}\right. \\
&\left.\quad-\sum_{i=1}^{n-1}\left(e^{z_{n-1, i}-x_{n, i}}+g_{i+1} e^{x_{n, i+1}-z_{n-1, i}}\right)-g_{n+1} e^{-x_{n n}-z_{n-1, n-1}}\right\}
\end{aligned}
$$

intertwines the Hamiltonian operators for the $D_{n}^{(1)}$ - and $C_{n-1}^{(1)}$-Toda chains:

$$
\begin{aligned}
\mathcal{H}^{D_{n}^{(1)}}\left(\underline{x}_{n}\right)= & -\frac{1}{2} \sum_{i=1}^{n} \frac{\partial^{2}}{\partial x_{n, i}^{2}}+g_{1} g_{2} e^{x_{n, 1}+x_{n}, 2} \\
& +\sum_{i=1}^{n-1} g_{i+1} e^{x_{n, i+1}-x_{n, i}}+g_{n+1} e^{-x_{n n}-x_{n, n-1}} \\
\mathcal{H}^{C_{n-1}^{(1)}}\left(\underline{z}_{n-1}\right)= & -\frac{1}{2} \sum_{i=1}^{n-1} \frac{\partial^{2}}{\partial z_{n-1, i}^{2}}+2 g_{1} e^{2 z_{n-1,1}} \\
& +\sum_{i=1}^{n-2} g_{i+1} e^{z_{n-1, i+1}-z_{n-1, i}}+2 g_{n} g_{n+1} e^{-2 z_{n-1, n-1}}
\end{aligned}
$$

We use the following notation for the integral kernels of the inverse transformations:

$$
\begin{aligned}
& Q_{C_{n}^{(1)}}^{D_{n+1}^{(1)}}\left(\underline{z}_{n+1}, \underline{x}_{n}\right):=Q_{D_{n+1}^{(1)}}^{C_{n}^{(1)}}\left(\underline{x}_{n}, \underline{z}_{n+1}\right), \\
& Q_{D_{n}^{(1)}}^{C_{n-1}^{(1)}}\left(\underline{z}_{n-1}, \underline{x}_{n}\right):=Q_{C_{n-1}^{(1)}}^{D_{n}^{(1)}}\left(\underline{x}_{n}, \underline{z}_{n-1}\right) .
\end{aligned}
$$

2.2.6. The integral operator with the kernel

$$
\begin{array}{r}
Q_{\widehat{B C}_{n+1}^{(2)}}^{D_{n}^{*}}\left(\underline{x}_{n}, \underline{z}_{n+1}\right)=\exp \left\{-g_{1} e^{z_{n+1,1}}-\sum_{i=1}^{n}\left(e^{x_{n, i}-z_{n+1, i}}+g_{i+1} e^{z_{n+1, i+1}-x_{n, i}}\right)\right. \\
\left.-g_{n+2} e^{-z_{n+1, n+1}}\right\}
\end{array}
$$

intertwines the Hamiltonian operators for the $D_{n}^{(2)}$ - and $\widehat{B C}_{n+1}$-Toda chains:

$$
\begin{aligned}
\mathcal{H}^{D_{n}^{(2)}}\left(\underline{x}_{n}\right)= & -\frac{1}{2} \sum_{i=1}^{n} \frac{\partial^{2}}{\partial x_{n, i}^{2}}+g_{1} e^{x_{1}}+\sum_{i=1}^{n-1} g_{i+1} e^{x_{n, i+1}-x_{n, i}}+g_{n+1} e^{-x_{n n}}, \\
\mathcal{H}^{\widehat{B C}_{n+1}^{*}\left(\underline{z}_{n+1}\right)=} & -\frac{1}{2} \sum_{i=1}^{n+1} \frac{\partial^{2}}{\partial z_{n+1, i}^{2}}-\frac{g_{1}}{2} e^{z_{n+1,1}}+\frac{g_{1}^{2}}{2} e^{2 z_{n+1,1}} \\
& +\sum_{i=1}^{n} g_{i+1} e^{z_{n+1, i+1}-z_{n+1, i}}-\frac{g_{n+2}}{2} e^{-z_{n+1, n+1}}+\frac{g_{n+2}^{2}}{2} e^{-2 z_{n+1, n+1}} .
\end{aligned}
$$

The integral kernel for the inverse transformation is denoted by

$$
Q_{D_{n}^{(2)}}^{\widehat{B C}_{n+1}^{*}}\left(\underline{z}_{n+1}, \underline{x}_{n}\right):=Q_{\widehat{B C}_{n+1}^{(2)}}^{D_{n}^{*}}\left(\underline{x}_{n}, \underline{z}_{n+1}\right) .
$$


Proposition 2.1. The integral operator with the kernel

$$
\begin{aligned}
& Q_{\widehat{I}_{n+1}}^{\widehat{B C}}\left(\underline{z}_{n+1} ; \underline{x}_{n}\right)=\left(\frac{1-e^{z_{n+1,1}}}{1+e^{z_{n+1,1}}}\right)^{\frac{g_{1}}{\sqrt{2 g_{2}}}}\left(\frac{1-e^{z_{n+1, n+1}}}{1+e^{z_{n+1, n+1}}}\right)^{\frac{g_{n+3}}{\sqrt{2 g_{n+2}}}}\left(\frac{1-e^{-2 z_{n+1, n+1}}}{1-e^{2 z_{n+1,1}}}\right)^{a} \\
& \times \exp \left\{a\left(\sum_{i=1}^{n+1} z_{n+1, i}-\sum_{k=1}^{n} x_{n, k}\right)\right.-\sqrt{\frac{g_{2}}{2}}\left(e^{x_{n, 1}+z_{n+1,1}}+e^{x_{n, 1}-z_{n+1,1}}\right) \\
&-\sum_{k=1}^{n-1} g_{k+2} e^{z_{n+1, k+1}-x_{n, k}}+e^{x_{n, k+1}-z_{n, k}} \\
&\left.-\sqrt{\frac{g_{n+2}}{2}}\left(e^{z_{n+1, n+1}-x_{n n}}+e^{-z_{n+1, n+1}-x_{n n}}\right)\right\}
\end{aligned}
$$

intertwines the quadratic Hamiltonians of the $\widehat{B C}_{n}$-Toda chain

$$
\begin{aligned}
H^{\widehat{B C}}\left(\underline{x}_{n}\right)= & -\frac{1}{2} \sum_{i=1}^{n} \frac{\partial^{2}}{\partial x_{n, i}^{2}}+g_{1} e^{x_{n, 1}}+g_{2} e^{2 x_{n, 1}} \\
& +\sum_{k=1}^{n-1} g_{k+2} e^{x_{n, k+1}-x_{n, k}}+g_{n+2} e^{-2 x_{n n}}-g_{n+3} e^{-x_{n n}}
\end{aligned}
$$

with the closed Inozemtsev quadratic Hamiltonian

$$
\begin{aligned}
H^{\widehat{I}_{n+1}}\left(\underline{z}_{n+1}\right)= & -\frac{1}{2} \sum_{i=1}^{n} \frac{\partial^{2}}{\partial z_{n+1, i}^{2}}+\frac{\widetilde{g}_{1}}{\left(e^{-z_{n+1,1} / 2}-e^{z_{n+1,1} / 2}\right)^{2}}+\frac{\widetilde{g}_{2}}{\left(e^{-z_{n+1,1}}-e^{z_{n+1,1}}\right)^{2}} \\
& +g_{3} \sqrt{\frac{g_{2}}{2}}\left(e^{z_{n+1,1}+z_{n+1,2}}+e^{z_{n+1,2}-z_{n+1,1}}\right)+\sum_{k=2}^{n-1} g_{k+2} e^{z_{n+1, k+1}-z_{n+1, k}} \\
& +\sqrt{\frac{g_{n+2}}{2}}\left(e^{z_{n+1,1}+z_{n+1,2}}+e^{z_{n+1,2}-z_{n+1,1}}\right)+\frac{a^{2}}{2} \\
& +\frac{\widetilde{g}_{n+3}}{\left(e^{-z_{n+1, n+1}}-e^{z_{n+1, n+1}}\right)^{2}}+\frac{\widetilde{g}_{n+4}}{\left(e^{-z_{n+1, n+1} / 2}-e^{z_{n+1, n+1} / 2}\right)^{2}}
\end{aligned}
$$

where

$$
\begin{aligned}
\tilde{g}_{1} & =-\frac{(2 a+1) g_{1}}{\sqrt{2 g_{2}}}, \quad \tilde{g}_{2}=2\left(a+\frac{g_{1}}{\sqrt{2 g_{2}}}\right)+2\left(a+\frac{g_{1}}{\sqrt{2 g_{2}}}\right)^{2}, \\
\tilde{g}_{n+3} & =2\left(a+\frac{g_{n+3}}{\sqrt{2 g_{n+2}}}\right)^{2}-2\left(a-\frac{g_{n+3}}{\sqrt{2 g_{n+2}}}\right), \quad \tilde{g}_{n+4}=(2 a-1) \frac{g_{n+3}}{\sqrt{2 g_{n+2}}} .
\end{aligned}
$$

2.3. $Q$-operators for affine Toda chains. Now we apply the results presented in the previous sections to the construction of the integral $Q$-operators for all classical series of affine Lie algebras as well as affine versions of the $B C_{n^{-}}$and $I_{n}$-Toda chains. We note that the elementary intertwining operators and recursive operators for finite Lie algebras can be obtained from the elementary intertwining operators and $\mathcal{Q}$-operators for affine Lie algebras by taking appropriate limits $g_{i} \rightarrow 0$ in (2.2)-(2.6). This generalizes the known relationship between the $\mathcal{Q}$-operator for $A_{n}^{(1)}$ and the recursive operators for $A_{n}$. 
Conjecture 2.1. The integral kernels for Q-operators have the following form:

$$
\begin{aligned}
& Q^{A_{2 n}^{(2)}}(\underline{x} ; \underline{y})=\int Q_{B C_{n+1}^{\prime}}^{A_{2 n}^{(2)}}\left(\underline{x} ; \underline{z}_{n+1}\right) Q_{A_{2 n}^{(2)}}^{B C_{n+1}^{\prime}}\left(\underline{z}_{n+1} ; \underline{y}\right) d \underline{z}_{n+1}, \\
& Q^{A_{2 n-1}^{(2)}}(\underline{x} ; \underline{y})=\int Q_{A_{2 n-1}^{(2)}}^{A_{(2)}^{(2)}}\left(\underline{x} ; \underline{z}_{n}\right) Q_{A_{2 n-1}^{(2)}}^{A_{(2)}^{(2)}}\left(\underline{z}_{n} \underline{y} ;\right) d \underline{z}_{n}, \\
& Q^{B_{n}^{(1)}}(\underline{x} ; \underline{y})=\int Q_{B C_{n}^{\prime \prime}}^{B_{B^{(1)}}}\left(\underline{x} ; \underline{z}_{n}\right) Q_{B_{n}^{(1)}}^{B C_{n}^{\prime \prime}}\left(\underline{z}_{n} ; \underline{y}\right) d \underline{z}_{n}, \\
& Q^{C_{n}^{(1)}}(\underline{x} ; \underline{y})=\int Q_{D_{n+1}^{(1)}}^{C_{n}^{(1)}}\left(\underline{x} ; \underline{z}_{n+1}\right) Q_{C_{n}^{(1)}}^{D_{n+1}^{(1)}}\left(\underline{z}_{n+1} ; \underline{y}\right) d \underline{z}_{n+1}, \\
& Q^{D_{n}^{(1)}}(\underline{x} ; \underline{y})=\int Q_{C_{n-1}^{(1)}}^{D_{n}^{(1)}}\left(\underline{x} ; \underline{z}_{n-1}\right) Q_{D_{n}^{(1)}}^{C_{n-1}^{(1)}}\left(\underline{z}_{n-1} ; \underline{y}\right) d \underline{z}_{n-1}, \\
& Q^{D_{n}^{(2)}}(\underline{x} ; \underline{y})=\int Q_{\widehat{B C}_{n+1}^{D_{n}^{(2)}}}\left(\underline{x} ; \underline{z}_{n+1}\right) Q_{D_{n}^{(2)}}^{\widehat{B C}_{n+1}}\left(\underline{z}_{n+1} ; \underline{y}\right) d \underline{z}_{n+1}, \\
& Q^{\widehat{B C}}(\underline{x} ; \underline{y})=\int Q_{\widehat{I}_{n+1}}^{\widehat{B C}_{n}}\left(\underline{x} ; \underline{z}_{n+1}\right) Q_{\widehat{B C}_{n+1}}^{\hat{B C}_{n}}\left(\underline{z}_{n+1} ; \underline{y}\right) d \underline{z}_{n+1} \text {, } \\
& Q^{\hat{I}_{n}}(\underline{x} ; \underline{y})=\int Q_{\widehat{B C}_{n-1}}^{\hat{I}_{n}}\left(\underline{x} ; \underline{z}_{n-1}\right) Q_{\widehat{I}_{n}}^{\widehat{B C}_{n-1}}\left(\underline{z}_{n-1} ; \underline{y}\right) d \underline{z}_{n-1},
\end{aligned}
$$

where $\underline{x}=\left(x_{1}, \ldots, x_{n}\right), \underline{y}=\left(y_{1}, \ldots, y_{n}\right)$, and $\underline{z}_{k}=\left(z_{k, 1}, \ldots, z_{k k}\right)$ for $k=n, n \pm 1$.

\section{§3. InTERTWINING OPERATORS FOR SEMI-INFINITE TODA CHAINS}

A similar approach can be applied to construct $Q$-operators for the infinite root systems $B_{\infty}, C_{\infty}, B C_{\infty}$, and $D_{\infty}$.

3.1. Quadratic Hamiltonians for semi-infinite Toda chains. Simple roots and Dynkin diagrams for the infinite Lie algebras $A_{\infty}, B_{\infty}, C_{\infty}, B C_{\infty}$, and $D_{\infty}$ are as follows:

$$
\begin{aligned}
& A_{\infty}: \quad \alpha_{i+1}=e_{i+1}-e_{i}, \quad i \in \mathbb{Z}, \\
& \cdots-\alpha_{-1}-\alpha_{0}-\alpha_{1}-\alpha_{2}-\cdots \text {; } \\
& B_{\infty}: \quad \alpha_{1}=e_{1}, \quad \alpha_{i+1}=e_{i+1}-e_{i}, \quad i \in \mathbb{Z}_{>0}, \\
& \alpha_{1} \Longleftarrow \alpha_{2}-\alpha_{3}-\cdots ; \\
& C_{\infty}: \quad \alpha_{1}=2 e_{1}, \quad \alpha_{i+1}=e_{i+1}-e_{i}, \quad i \in \mathbb{Z}_{>0}, \\
& \alpha_{1} \Longrightarrow \alpha_{2} \longrightarrow \alpha_{3} \longrightarrow \cdots ; \\
& D_{\infty}: \quad \alpha_{1}=e_{1}+e_{2}, \quad \alpha_{i+1}=e_{i+1}-e_{i}, \quad i \in \mathbb{Z}_{>0}, \\
& \alpha_{1} \\
& \alpha_{3}{ }^{\alpha_{1}}-\alpha_{4}-\cdots ; \\
& \alpha_{2}
\end{aligned}
$$




$$
B C_{\infty}: \quad \alpha_{0}=2 e_{1}, \quad \alpha_{1}=e_{1}, \quad \alpha_{i+1}=e_{i+1}-e_{i}, \quad i \in \mathbb{Z}_{>0},
$$

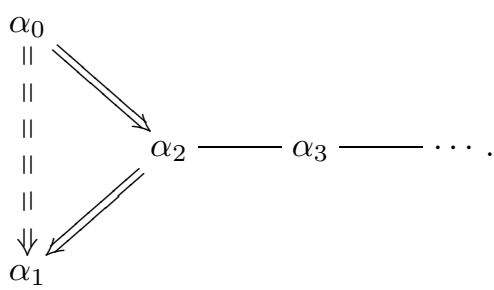

3.2. Elementary intertwiners for semi-infinite Toda chains. The $Q$-operator for the $A_{\infty}$-Toda chain is known (see [T] for the classical limit) and has an elementary structure. Namely, it is given by the integral operator with the kernel

$$
Q(\underline{x}, \underline{y})=\exp \left\{-\sum_{i \in \mathbb{Z}}\left(e^{x_{i}-y_{i}}+g_{i} e^{y_{i+1}-x_{i}}\right)\right\}
$$

and intertwines the $A_{\infty}$-Toda chain Hamiltonians

$$
\begin{aligned}
& \mathcal{H}^{A_{\infty}}(\underline{x})=-\frac{1}{2} \sum_{i \in \mathbb{Z}} \frac{\partial^{2}}{\partial x_{i}^{2}}+\sum_{i \in \mathbb{Z}} g_{i} e^{x_{i+1}-x_{i}}, \\
& \mathcal{H}^{A_{\infty}}(\underline{y})=-\frac{1}{2} \sum_{i \in \mathbb{Z}} \frac{\partial^{2}}{\partial y_{i}^{2}}+\sum_{i \in \mathbb{Z}} g_{i} e^{y_{i+1}-y_{i}} .
\end{aligned}
$$

In this section the notation $\underline{x}=\left(x_{1}, x_{2}, \ldots\right), \underline{y}=\left(y_{1}, y_{2}, \ldots\right)$ is used. Below we conjecture a generalization of this integral operator to other classical series as well as to the $B C$ and $I$-series of Toda chains. The intertwining relations with quadratic Hamiltonians can be checked straightforwardly.

3.2.1. The integral operator with the kernel

$$
Q_{B C_{\infty}^{*}}^{B_{\infty}}(\underline{x}, \underline{z})=\exp \left\{-g_{1} e^{z_{1}}-\sum_{i>0}\left(e^{x_{i}-z_{i}}+g_{i+1} e^{z_{i+1}-x_{i}}\right)\right\},
$$

intertwines the $B_{\infty^{-}}$and $B C_{\infty}^{*}$-Toda chain Hamiltonian operators

$$
\begin{aligned}
\mathcal{H}^{B}(\underline{x}) & =-\frac{1}{2} \sum_{i=1}^{\infty} \frac{\partial^{2}}{\partial x_{i}^{2}}+g_{1} e^{x_{1}}+\sum_{i=1}^{\infty} g_{i+1} e^{x_{i+1}-x_{i}} \\
\mathcal{H}^{B C_{\infty}^{*}}(\underline{z}) & =-\frac{1}{2} \sum_{i=1}^{\infty} \frac{\partial^{2}}{\partial z_{i}^{2}}-\frac{g_{1}}{2} e^{z_{1}}+\frac{g_{1}^{2}}{2} e^{2 z_{1}}+\sum_{i=1}^{\infty} g_{i+1} e^{z_{i+1}-z_{i}} .
\end{aligned}
$$

3.2.2. Similarly, the integral operator with the kernel

$$
Q_{D_{\infty}}^{C_{\infty}}(\underline{x}, \underline{z})=\exp \left\{-g_{1} e^{x_{1}+z_{1}}-\sum_{i>0}\left(e^{x_{i}-z_{i}}+g_{i+1} e^{z_{i+1}-x_{i}}\right)\right\}
$$

intertwines the $C_{\infty^{-}}$and $D_{\infty}$-Toda chain Hamiltonian operators

$$
\begin{aligned}
& \mathcal{H}^{C_{\infty}}(\underline{x})=-\frac{1}{2} \sum_{i=1}^{\infty} \frac{\partial^{2}}{\partial x_{i}^{2}}+2 g_{1} e^{2 x_{1}}+\sum_{i=1}^{\infty} g_{i+1} e^{x_{i+1}-x_{i}}, \\
& \mathcal{H}^{D_{\infty}}(\underline{z})=-\frac{1}{2} \sum_{i=1}^{\infty} \frac{\partial^{2}}{\partial z_{i}^{2}}+g_{1} g_{2} e^{z_{1}+z_{2}}+\sum_{i=1}^{\infty} g_{i+1} e^{z_{i+1}-z_{i}} .
\end{aligned}
$$


Thus, the integral $Q$-operator for $C_{\infty}$ has the kernel

$$
Q^{C_{\infty}}(\underline{x} ; \underline{y})=\int Q_{D_{\infty}}^{C_{\infty}}(\underline{x}, \underline{z}) \cdot Q_{C_{\infty}^{\infty}}^{D_{\infty}}(\underline{z}, \underline{y}) \prod_{i=1}^{\infty} d z_{i} .
$$

3.2.3. The integral operator with the kernel

$$
Q_{C_{\infty}}^{D_{\infty}}(\underline{z}, \underline{x})=\exp \left\{-g_{1} e^{x_{1}+z_{1}}-\sum_{i>0}\left(e^{z_{i}-x_{i}}+g_{i+1} e^{x_{i+1}-z_{i}}\right)\right\}
$$

intertwines the $D_{\infty}$ - and $C_{\infty}$-Toda chain Hamiltonian operators

$$
\begin{aligned}
& \mathcal{H}^{D_{\infty}}(\underline{x})=-\frac{1}{2} \sum_{i=1}^{\infty} \frac{\partial^{2}}{\partial x_{i}^{2}}+g_{1} g_{2} e^{x_{1}+x_{2}}+\sum_{i=1}^{\infty} g_{i+1} e^{x_{i+1}-x_{i}} \\
& \mathcal{H}^{C_{\infty}}\left(z_{i}\right)=-\frac{1}{2} \sum_{i=1}^{\infty} \frac{\partial^{2}}{\partial z_{i}^{2}}+2 g_{1} e^{2 z_{1}}+\sum_{i=1}^{\infty} g_{i+1} e^{z_{i+1}-z_{i}}
\end{aligned}
$$

3.2.4. Consider the quadratic Hamiltonian of the $B C_{\infty}$-Toda chain with arbitrary coupling constants:

$$
\mathcal{H}^{B C_{\infty}}(\underline{x})=-\frac{1}{2} \sum_{i=1}^{\infty} \frac{\partial^{2}}{\partial x_{i}^{2}}+g_{1} e^{x_{1}}+g_{2} e^{2 x_{1}}+\sum_{i=1}^{\infty} g_{i+2} e^{x_{i+1}-x_{i}} .
$$

Then the integral operator with the kernel

$$
\begin{aligned}
Q_{I_{\infty}}^{B C_{\infty}}(\underline{x} ; \underline{z})= & \left(1+e^{z_{n, 1}}\right)^{-\frac{g_{1}}{\sqrt{2 g_{2}}}-a}\left(1-e^{z_{n, 1}}\right)^{\frac{g_{1}}{\sqrt{2 g_{2}}}-a} \\
& \times \exp \left\{a \sum_{i>0}\left(z_{n, i}-x_{n, i}\right)-\sqrt{\frac{g_{2}}{2}}\left(e^{x_{1}+z_{1}}+e^{x_{1}-z_{1}}\right)\right. \\
& \left.-\sum_{i>0}\left(g_{i+1} e^{z_{i+1}-x_{i}}+e^{x_{i+1}-z_{i+1}}\right)\right\}
\end{aligned}
$$

intertwines $\mathcal{H}^{B C_{\infty}}(\underline{x})$ with the following specific quadratic Hamiltonian of the $I_{\infty}$-integrable system:

$$
\begin{aligned}
H^{I_{\infty}}(\underline{z})= & -\frac{1}{2} \sum_{i=1}^{\infty} \frac{\partial^{2}}{\partial z_{i}^{2}}+\frac{\widetilde{g}_{1}}{\left(e^{-z_{1} / 2}-e^{z_{1} / 2}\right)^{2}}+\frac{\widetilde{g}_{2}}{\left(e^{-z_{1}}-e^{z_{1}}\right)^{2}} \\
& +\sqrt{\frac{g_{2}}{2}} e^{z_{1}+z_{2}}+g_{3} \sqrt{\frac{g_{2}}{2}} e^{z_{2}-z_{1}}+\sum_{i=2}^{\infty} g_{i+2} e^{z_{i+1}-z_{i}}
\end{aligned}
$$

where the coupling constants $\widetilde{g}_{1}$ and $\widetilde{g}_{2}$ are given by (1.31):

$$
\tilde{g}_{1}=-\frac{(2 a+1) g_{1}}{\sqrt{2 g_{2}}}, \quad \tilde{g}_{2}=2\left(a+\frac{g_{1}}{\sqrt{2 g_{2}}}\right)+2\left(a+\frac{g_{1}}{\sqrt{2 g_{2}}}\right)^{2} .
$$

We denote by $Q_{B C_{\infty}}^{I_{\infty}}(\underline{z} ; \underline{x})$ the kernel of the inverse integral transformation.

3.3. $Q$-operators for semi-infinite Toda chains. Now we can construct $Q$-operators for the semi-infinite Toda chains, combining the elementary intertwiners obtained in previous subsections. 
Conjecture 3.1. The Q-operators for semi-infinite Toda chains are given by the following integral kernels:

$$
\begin{aligned}
Q^{B_{\infty}}(\underline{x} ; \underline{y}) & =\int Q_{B C_{\infty}}^{B_{\infty}}\left(x_{i}, z_{i}\right) \cdot Q_{B_{\infty}}^{B C_{\infty}}\left(z_{i}, y_{i}\right) \prod_{i=1}^{\infty} d z_{i} ; \\
Q^{C_{\infty}}(\underline{x} ; \underline{y}) & =\int Q_{D_{\infty}}^{C_{\infty}}(\underline{x}, \underline{z}) \cdot Q_{C_{\infty}^{\infty}}^{D_{\infty}}(\underline{z}, \underline{y}) \prod_{i=1}^{\infty} d z_{i} ; \\
Q^{D_{\infty}}(\underline{x} ; \underline{y}) & =\int Q_{C_{\infty}}^{D_{\infty}}\left(x_{i}, z_{i}\right) \cdot Q_{D_{\infty}}^{C_{\infty}}\left(z_{i}, y_{i}\right) \prod_{i=1}^{\infty} d z_{i} ; \\
Q^{B C_{\infty}}(\underline{x} ; \underline{y}) & =\int Q_{I_{\infty}}^{B C_{\infty}}\left(x_{i}, z_{i}\right) \cdot Q_{B C_{\infty}}^{I_{\infty}}\left(z_{i}, y_{i}\right) \prod_{i=1}^{\infty} d z_{i} ; \\
Q^{I_{\infty}}(\underline{z} ; \underline{y}) & =\int Q_{B C_{\infty}}^{I_{\infty}}\left(z_{i}, x_{i}\right) \cdot Q_{I_{\infty}}^{B C_{\infty}}\left(x_{i}, y_{i}\right) \prod_{i=1}^{\infty} d x_{i} .
\end{aligned}
$$

One can directly check this conjecture for the intertwining relations with quadratic Hamiltonians.

\section{REFERENCES}

[F] L. D. Faddeev, How the algebraic Bethe ansatz works for integrable models, Symétries Quantiques (Les Houches, 1995), North-Holland, Amsterdam, 1998, pp. 149-219. MR1616371 (2000b:82010)

[GKLO] A. Gerasimov, S. Kharchev, D. Lebedev, and S. Oblezin, On a Gauss-Givental representation for quantum Toda chain wave function, Int. Math. Res. Not. 2006, Art. ID 96489; arXiv:math.RT/0505310. MR2219213 (2007f:17041)

[GLO1] A. Gerasimov, D. Lebedev, and S. Oblezin, Givental integral representation for classical groups, arXiv:math. RT/ 0608152.

[GLO2] - New integral representations of Whittaker functions for classical groups, math.RT/ 0705.2886.

[GLO3] _ Baxter operator and Archimedean Hecke algebra, Comm. Math. Phys. 284 (2008), 867896; DOI 10.1007/s00220-008-0547-9, arXiv:math.RT/0706.3476. MR2452597(2009k:17049)

[Gi] A. Givental, Stationary phase integrals, quantum Toda lattices, flag manifolds and the mirror conjecture, Topics in Singularity Theory, Amer. Math. Soc. Transl. Ser. 2, vol. 180, Amer. Math. Soc., Providence, RI, 1997, pp. 103-115; arXiv:alg-geom/9612001. MR.1767115 (2001d:14063)

[Ha] M. Hashizume, Whittaker functions on semi-simple Lie groups, Hiroshima Math. J. 12 (1982), 259-293. MR.0665496 (84d:22018)

[He] S. Helgason, Differential geometry, Lie groups, and symmetric spaces, Pure Appl. Math., vol. 80, Acad. Press, Inc., New York-London, 1978. MR0514561 (80k:53081)

[I] V. Inozemtsev, The finite Toda lattices, Comm. Math. Phys. 121 (1989), 629-638. MR0990995 (90f:58086)

[K] V. Kac, Infinite-dimensional Lie algebras, Cambridge University Press, Cambridge, 1990. MR.1104219(92k:17038)

[KS] V. Kuznetsov and E. K. Sklyanin, Bäcklund transformation for the BC-type Toda lattice, Vadim Kuznetsov Memorial Issue on Integrable Systems and Related Topics, SIGMA Symmetry Integrability Geom. Methods Appl. 3 (2007), 080, 17 pp.; arXiv:0707.1950. MR2366942 (2009j:70024)

[PG] V. Pasquier and M. Gaudin, The periodic Toda chain and a matrix generalization of the Bessel function recursion relations, J. Phys. A 25 (1992), 5243-5252. MR1192958 (94a:82023)

[RSTS] A. G. Reuman and M. A. Semenov-Tyan-Shanskiǔ, Integrable systems. Group-theoretical approach, Inst. Kompyuter. Issled., Moscow-Izhevsk, 2003. (Russian)

[S] E. K. Sklyanin, Boundary conditions for integrable quantum systems, J. Phys. A 21 (1988), 2375-2389. MR0953215 (89h:81258) 
[STS] Dynamical Systems-7, Itogi Nauki i Tekhniki. Sovrem. Probl. Mat. Fundam. Naprav., vol. 16, VINITI, Moscow, 1987; English transl., Encyclopaedia Math. Sci., vol. 16, Springer-Verlag, Berlin, 1994, pp. 226-259. MR0922069 (88g:58004)

[T] M. Toda, Theory of nonlinear lattices, Springer Ser. in Solid-State Sci., vol. 20, Springer-Verlag, Berlin-New York, 1981. MR0618652 (82k:58052b)

Institute for Theoretical and Experimental Physics, Moscow 117259, Russia; School of Mathematics, Trinity College, Dublin 2, Ireland; and Hamilton Mathematics Institute, Trinity College, Dublin 2, Ireland

E-mail address: anton@maths.tcd.ie

Institute for Theoretical and Experimental Physics, Moscow 117259, Russia

E-mail address: lebedev@itep.ru

Institute for Theoretical and Experimental Physics, Moscow 117259, Russia

E-mail address: Sergey.Oblezin@itep.ru

Received 11/JAN/2010

Originally published in English 